\title{
Second and third orders asymptotic expansions for the distribution of particles in a branching random walk with a random environment in time
}

\author{
ZHIQIANG GAO ${ }^{1}$ and QUANSHENG LIU ${ }^{2,3}$ \\ ${ }^{1}$ School of Mathematical Sciences, Laboratory of Mathematics and Complex Systems, Beijing Normal Uni- \\ versity, Beijing 100875, P. R. China. E-mail: gaozq@bnu.edu.cn \\ ${ }^{2}$ Université de Bretagne-Sud, CNRS UMR 6205, LMBA, campus de Tohannic, F-56000 Vannes, France. \\ E-mail: quansheng.liu@univ-ubs.fr \\ ${ }^{3}$ Changsha University of Science and Technology, School of Mathematics and Statistics, Changsha 410004, \\ P. R. China
}

Consider a branching random walk in which the offspring distribution and the moving law both depend on an independent and identically distributed random environment indexed by the time. For the normalised counting measure of the number of particles of generation $n$ in a given region, we give the second and third orders asymptotic expansions of the central limit theorem under rather weak assumptions on the moments of the underlying branching and moving laws. The obtained results and the developed approaches shed light on higher order expansions. In the proofs, the Edgeworth expansion of central limit theorems for sums of independent random variables, truncating arguments and martingale approximation play key roles. In particular, we introduce a new martingale, show its rate of convergence, as well as the rates of convergence of some known martingales, which are of independent interest.

Keywords: asymptotic expansion; branching random walks; central limit theorem; convergence rate; martingale approximation; random environment

\section{Introduction}

A central limit theorem for the branching random walk has been initiated and conjectured by Harris ([23], Chapter III, Section 16). Since then this conjecture has been proved in various forms and for various models, see, for example, [2,7,19,27,29,30,33,39,42]. For the special cases where the underlying motion law is governed by the Wiener process or the simple symmetric walk, Révész [35] investigated the speed of convergence in the central limit theorem and conjectured the exact convergence rate, which was confirmed by Chen [11] and complemented by Gao [20]. Kabluchko [28] recovered and generalized Chen's results by using a general approach. Gao and Liu [17] improved and extended Chen's results on the branching Wiener process to the strongly non-lattice case under much weaker moment conditions. Révész, Rosen and Shi [36] found full asymptotic expansions in the local limit theorem for branching Wiener processes, while Grübel and Kabluchko [22] obtained the similar result for a branching random walk on $\mathbb{Z}$ and discussed 
the related applications in random trees. The exact convergence rate obtained in $[11,17]$ can be formulated as the first order asymptotic expansion in the central limit theorem for the models considered therein. Inspired by these works, we consider the following natural question: what about the asymptotic expansion of higher orders?

The aim of this article is to derive the second and third orders asymptotic expansions in the central limit theorem for a branching random walk with a time-dependent random environment. The goal is twofold. On the one hand, although central limit theorems for branching random walks have been well studied and the asymptotic expansions for branching Wiener processes and lattice branching random walks were given in [36] and [22], the asymptotic expansions in central limit theorems for non-lattice branching random walks are still not known. On the other hand, we shall perform our research in a more general framework, that is, for a branching random walk with a random environment in time, which is a natural generalization of classical branching random walk formulated in Harris [23]. This model first appeared in Biggins [8] as a particular case of a general framework, and more related limit theorems were given in [25,32,41]. For other different kinds of branching random walks in random environments, the reader may refer to $[6,9,10,12-14,16,21,24,33,42]$. For other different aspects on branching random walks, see [37] and [43].

This article opens the way to obtain higher order asymptotic expansions. The second and third orders expansions given here serve as good examples. The obtained results and the developed methods can be used to obtain asymptotic expansions of orders 4,5 , etc., and hint the general formula for each finite order expansion, although we have not yet been able to prove it: see Conjecture 2.7 and the comments following it. We also mention that the approaches in our previous work [17] have been significantly developed in the present article.

The article is organized as follows. In Section 2, after giving the rigorous definition of the model of a branching random walk with a random environment in time and introducing three martingales, we formulate the results on convergence rates of martingales as Theorem 2.1, and then state the main results on the asymptotic expansions in Theorems 2.3 and 2.4. Section 3 presents some preliminaries including a result on the Edgeworth expansion for the distribution function of sums of independent random variables and a key decomposition used in the proofs. Section 4 is devoted to the proofs of main results. While the proof of Theorem 2.1 is postponed to Section 5 .

\section{Main results}

\subsection{Description of the model}

The model a branching random walk with a random environment in time can be formulated as follows $[17,19]$. Let $(\Theta, \mathbb{P})$ be a probability space, and $\left(\Theta^{\mathbb{N}}, \mathbb{p}^{\otimes \mathbb{N}}\right)=(\Omega, \tau)$ be the corresponding product space. For a sequence $\xi \in \Omega$, we denote $\xi=\left(\xi_{1}, \xi_{2}, \ldots\right)$, where $\xi_{k}$ are the $k$ th coordinate function on $\Omega$. Then $\xi=\left(\xi_{n}\right)$ will serve as an independent and identically distributed environment. Let $\theta$ be the usual shift transformation on $\Theta^{\mathbb{N}}: \theta\left(\xi_{0}, \xi_{1}, \ldots\right)=\left(\xi_{1}, \xi_{2}, \ldots\right)$. To each realization of $\xi_{n}$ correspond two probability distributions: the offspring distribution $p\left(\xi_{n}\right)=\left(p_{0}\left(\xi_{n}\right), p_{1}\left(\xi_{n}\right), \ldots\right)$ on $\mathbb{N}=\{0,1, \ldots\}$, and the moving distribution $G\left(\xi_{n}\right)$ on $\mathbb{R}$. 
Given the environment $\xi=\left(\xi_{n}\right)$, the branching random walk in varying environment evolves according to the following rules:

- At time 0 , an initial particle $\varnothing$ of generation 0 is located at the origin $S_{\varnothing}=0$.

- At time $1, \varnothing$ is replaced by $N=N_{\varnothing}$ new particles of generation 1 , and for $1 \leq i \leq N$, each particle $\varnothing i$ moves to $S_{\varnothing i}=S_{\varnothing}+L_{i}$, where $N, L_{1}, L_{2}, \ldots$ are mutually independent, $N$ has the law $p\left(\xi_{0}\right)$, and each $L_{i}$ has the law $G\left(\xi_{0}\right)$.

- At time $n+1$, each particle $u=u_{1} u_{2} \cdots u_{n}$ of generation $n$ is replaced by $N_{u}$ new particles of generation $n+1$, with displacements $L_{u 1}, L_{u 2}, \ldots, L_{u N_{u}}$. That means for $1 \leq i \leq N_{u}$, each particle $u i$ moves to $S_{u i}=S_{u}+L_{u i}$, where $N_{u}, L_{u 1}, L_{u 2}, \ldots$ are mutually independent, $N_{u}$ has the law $p\left(\xi_{n}\right)$, and each $L_{u i}$ has the same law $G\left(\xi_{n}\right)$. We do not assume the independence between $p\left(\xi_{n}\right)$ and $G\left(\xi_{n}\right), n \geq 0$.

By definition, given the environment $\xi$, the random variables $N_{u}$ and $L_{u}$, indexed by all the finite sequences $u$ of positive integers, are independent of each other. For each realization $\xi \in \Theta^{\mathbb{N}}$ of the environment sequence, let $\left(\Gamma, \mathcal{G}, \mathbb{P}_{\xi}\right)$ be the probability space on which the process is defined (when the environment $\xi$ is fixed to the given realization). The probability $\mathbb{P}_{\xi}$ is usually called quenched law. The total probability space can be formulated as the product space $\left(\Theta^{\mathbb{N}} \times \Gamma, \mathcal{E}^{\mathbb{N}} \otimes\right.$ $\mathcal{G}, \mathbb{P})$, where $\mathbb{P}=\mathbb{E}\left(\delta_{\xi} \otimes \mathbb{P}_{\xi}\right)$ with $\delta_{\xi}$ the Dirac measure at $\xi$ and $\mathbb{E}$ the expectation with respect to the random variable $\xi$, so that for all measurable and positive $g$ defined on $\Theta^{\mathbb{N}} \times \Gamma$, we have

$$
\int_{\Theta^{\mathbb{N}} \times \Gamma} g(x, y) d \mathbb{P}(x, y)=\mathbb{E} \int_{\Gamma} g(\xi, y) d \mathbb{P}_{\xi}(y) .
$$

The total probability $\mathbb{P}$ is usually called annealed law. The quenched law $\mathbb{P}_{\xi}$ may be considered to be the conditional probability of $\mathbb{P}$ given $\xi$. The expectation with respect to $\mathbb{P}$ will still be denoted by $\mathbb{E}$; there will be no confusion for reason of consistence. The expectation with respect to $\mathbb{P}_{\xi}$ will be denoted by $\mathbb{E}_{\xi}$.

Let $\mathbb{T}$ be the genealogical tree with $\left\{N_{u}\right\}$ as defining elements. By definition, we have: (a) $\varnothing \in$ $\mathbb{T}$; (b) $u i \in \mathbb{T}$ implies $u \in \mathbb{T}$; (c) if $u \in \mathbb{T}$, then $u i \in \mathbb{T}$ if and only if $1 \leq i \leq N_{u}$. Let $\mathbb{T}_{n}=\{u \in$ $\mathbb{T}:|u|=n\}$ be the set of particles of generation $n$, where $|u|$ denotes the length of the sequence $u$ and represents the number of generation to which $u$ belongs.

\subsection{The main results}

Let $Z_{n}(\cdot)$ be the counting measure of particles of generation $n$ : for $B \subset \mathbb{R}$,

$$
Z_{n}(B)=\sum_{u \in \mathbb{T}_{n}} \mathbf{1}_{B}\left(S_{u}\right)
$$

Then $\left\{Z_{n}(\mathbb{R})\right\}$ constitutes a branching process in a random environment (see, e.g., [3,4,38]). For $n \geq 0$, let $1_{n}=(1, \ldots, 1)$ be the sequence of $n$ times 1 , with the convention that $1_{0}=\varnothing$, and set $\widehat{N}_{n}=N_{1_{n}}$ (resp. $\left.\widehat{L}_{n}=L_{1_{n+1}}\right)$, whose distribution under $\mathbb{P}_{\xi}$ is the common one $p\left(\xi_{n}\right)$ (resp. $\left.G\left(\xi_{n}\right)\right)$ of each $N_{u}$ (resp. $L_{u i}, i \geq 1$ ) with $|u|=n$, and define

$$
m_{n}=m\left(\xi_{n}\right)=\mathbb{E}_{\xi} \widehat{N}_{n}, \quad \Pi_{n}=m_{0} \cdots m_{n-1}, \quad \Pi_{0}=1 .
$$


It is well known that the normalized sequence

$$
W_{n}=\Pi_{n}^{-1} Z_{n}(\mathbb{R}), \quad n \geq 1
$$

constitutes a martingale with respect to the filtration $\mathcal{F}_{n}$ defined by:

$$
\mathcal{F}_{0}=\{\varnothing, \Omega\}, \quad \mathcal{F}_{n}=\sigma\left(\xi, N_{u}:|u|<n\right), \quad \text { for } n \geq 1 .
$$

Throughout the article, we shall always assume the following conditions:

$$
\mathbb{E} \ln m_{0}>0 \text { and } \mathbb{E}\left(\frac{1}{m_{0}} \widehat{N}_{0}\left(\ln \widehat{N}_{0}\right)^{1+\lambda}\right)<\infty,
$$

where the value of $\lambda>0$ will be specified in the hypothesis of theorems, and $\ln ^{+} x=\max (\ln x, 0)$ (resp. $\ln ^{-} x=\max (-\ln x, 0)$ ) denotes the positive (resp. negative) part of $\ln x$ for $x>0$. It is well known that the limit

$$
W=\lim _{n} W_{n}
$$

exists almost surely (a.s.) by the martingale convergence theorem, and that, under (2.1), $\mathbb{E} W=1$ and $W>0$ a.s. on the explosion event $\left\{Z_{n}(\mathbb{R}) \rightarrow \infty\right\}$ (in fact (2.1) with $\lambda=0$ suffices for these assertions: see [4] and [40]). In particular, the underlying branching process is supercritical and $Z_{n}(\mathbb{R}) \rightarrow \infty$ with positive probability.

For $n \geq 0$, define

$$
\begin{aligned}
l_{n} & =\mathbb{E}_{\xi} \widehat{L}_{n}, \quad \sigma_{n}^{(v)}=\mathbb{E}_{\xi}\left(\widehat{L}_{n}-l_{n}\right)^{\nu} \quad \text { for } v \geq 2 \\
\ell_{n} & =\sum_{k=0}^{n-1} l_{k}, \quad s_{n}^{(v)}=\sum_{k=0}^{n-1} \sigma_{k}^{(v)} \quad \text { for } v \geq 2, \quad s_{n}=\left(s_{n}^{(2)}\right)^{1 / 2} .
\end{aligned}
$$

Since $\left\{\xi_{n}\right\}$ are i.i.d., by the law of large numbers, we see that

$$
s_{n}^{(v)} \sim n \mathbb{E} \sigma_{0}^{(v)},
$$

where $a_{n} \sim b_{n}$ means $\lim _{n \rightarrow \infty} a_{n} / b_{n}=1$. This will be frequently used later.

To state our main result, we shall need the following martingales:

$$
\begin{aligned}
& N_{1, n}=\frac{1}{\Pi_{n}} \sum_{u \in \mathbb{T}_{n}}\left(S_{u}-\ell_{n}\right), \\
& N_{2, n}=\frac{1}{\Pi_{n}} \sum_{u \in \mathbb{T}_{n}}\left[\left(S_{u}-\ell_{n}\right)^{2}-s_{n}^{2}\right], \\
& N_{3, n}=\frac{1}{\Pi_{n}} \sum_{u \in \mathbb{T}_{n}}\left[\left(S_{u}-\ell_{n}\right)^{3}-3\left(S_{u}-\ell_{n}\right) s_{n}^{2}-s_{n}^{(3)}\right],
\end{aligned}
$$


with respect to the filtration $\left(\mathscr{D}_{n}\right)$ defined by

$$
\mathscr{D}_{0}=\{\varnothing, \Omega\}, \quad \mathscr{D}_{n}=\sigma\left(\xi, N_{u}, L_{u i}: i \geq 1,|u|<n\right) \quad \text { for } n \geq 1 .
$$

Theorem 2.1 (Convergence rates of the martingales). The sequences $\left\{\left(N_{v, n}, D_{n}\right)\right\}(v=$ $1,2,3)$ are martingales. Moreover, we have the following assertions about their rate of convergence:

(1) Assume (2.1) and $\mathbb{E}\left(\ln ^{-} m_{0}\right)^{1+\lambda}<\infty$ for some $\lambda>1$, together with $\mathbb{E}\left(\left|\widehat{L}_{0}\right|^{\eta}\right)<\infty$ for some $\eta>2$. Then there exists a real random variable $V_{1}$ such that a.s.

$$
N_{1, n}-V_{1}=o\left(n^{-\lambda+1+\delta}\right) \quad \forall \delta>0 .
$$

(2) Assume (2.1) and $\mathbb{E}\left(\ln ^{-} m_{0}\right)^{1+\lambda}<\infty$ for some $\lambda>2$, together with $\mathbb{E}\left(\left|\widehat{L}_{0}\right|^{\eta}\right)<\infty$ for some $\eta>4$. Then there exists a real random variable $V_{2}$ such that a.s.

$$
N_{2, n}-V_{2}=o\left(n^{-\lambda+2+\delta}\right) \quad \forall \delta>0 .
$$

(3) Assume (2.1) and $\mathbb{E}\left(\ln ^{-} m_{0}\right)^{1+\lambda}<\infty$ for some $\lambda>3$, together with $\mathbb{E}\left(\left|\widehat{L}_{0}\right|^{\eta}\right)<\infty$ for some $\eta>6$. Then there exists a real random variable $V_{3}$ such that a.s.

$$
N_{3, n}-V_{3}=o\left(n^{-\lambda+3+\delta}\right) \quad \forall \delta>0 .
$$

The proof is postponed to Section 5 .

Remark 2.2. A weaker version of parts (1) and (2) has been proved in [17], Propositions 2.1 and 2.2, where the convergence of the martingales $\left(N_{1, n}\right)$ and $\left(N_{2, n}\right)$ was shown under the same conditions. The martingale $\left(N_{3, n}\right)$ appears for the first time in this article.

For asymptotic expansions of the central limit theorem, we will need the following hypotheses on the motion law $G\left(\xi_{0}\right)$ of particles:

$$
\mathbb{P}\left(\limsup _{|t| \rightarrow \infty}\left|\mathbb{E}_{\xi} e^{i t \widehat{L}_{0}}\right|<1\right)>0 \quad \text { and } \quad \mathbb{E}\left(\left|\widehat{L}_{0}\right|^{\eta}\right)<\infty
$$

where the value of $\eta>1$ will be specified in the theorems. The first hypothesis means that Cramér's condition about the characteristic function of $G\left(\xi_{0}\right)$ holds with positive probability. Set

$$
Z_{n}(t)=Z_{n}((-\infty, t]), \quad \phi(t)=\frac{1}{\sqrt{2 \pi}} e^{-t^{2} / 2}, \quad \Phi(t)=\int_{-\infty}^{t} \phi(x) d x, \quad t \in \mathbb{R} .
$$

Denote by $H_{m}(\cdot)$ the Chebyshev-Hermite polynomial of degree $m$ :

$$
H_{m}(x)=m ! \sum_{k=0}^{\left\lfloor\frac{m}{2}\right\rfloor} \frac{(-1)^{k} x^{m-2 k}}{k !(m-2 k) ! 2^{k}}
$$


where $\lfloor x\rfloor$ denotes the largest integer not bigger than $x$. More precisely, we need the following polynomials:

$$
\begin{aligned}
& H_{0}(x)=1, \quad H_{1}(x)=x, \quad H_{2}(x)=x^{2}-1, \quad H_{3}(x)=x^{3}-3 x \\
& H_{4}(x)=x^{4}-6 x^{2}+3, \quad H_{5}(x)=x^{5}-10 x^{3}+15 x \\
& H_{6}(x)=x^{6}-15 x^{4}+45 x^{2}-15, \quad H_{8}(x)=x^{8}-28 x^{6}+210 x^{4}-420 x^{2}+105 .
\end{aligned}
$$

In [17], Theorem 2.3, the authors proved the following result about the exact rate of convergence in the central limit theorem: if $\mathbb{E} m_{0}^{-\delta}<\infty$ for some $\delta>0,(2.1)$ holds for some $\lambda>8$ and (2.2) holds for some $\eta>12$, then for all $t \in \mathbb{R}$,

$$
\sqrt{n}\left[\frac{1}{\Pi_{n}} Z_{n}\left(\ell_{n}+s_{n} t\right)-\Phi(t) W\right] \stackrel{n \rightarrow \infty}{\longrightarrow}-\frac{\phi(t) V_{1}}{\left(\mathbb{E} \sigma_{0}^{(2)}\right)^{1 / 2}}-\frac{\left(\mathbb{E} \sigma_{0}^{(3)}\right) H_{2}(t) \phi(t) W}{6\left(\mathbb{E} \sigma_{0}^{(2)}\right)^{3 / 2}} \quad \text { a.s. }
$$

From this result we can deduce the following version describing the first order expansion in the central limit theorem: for $t \in \mathbb{R}$, as $n \rightarrow \infty$,

$$
\frac{1}{\Pi_{n}} Z_{n}\left(\ell_{n}+s_{n} t\right)=\left(\Phi(t)+\frac{Q_{1, n}(t)}{n^{1 / 2}}\right) W+\left(-\frac{1}{s_{n}}\right) \phi(t) V_{1}+o\left(\frac{1}{\sqrt{n}}\right) \quad \text { a.s. }
$$

where

$$
\frac{Q_{1, n}(t)}{n^{1 / 2}}=-\frac{s_{n}^{(3)}}{6 s_{n}^{3}} H_{2}(t) \phi(t) .
$$

In this article, we are interested in higher order expansions. Our main results are the following two theorems about the second and third orders expansions in the central limit theorem. Naturally, for a higher order expansion, we need higher order moment conditions.

Theorem 2.3 (Second order expansion). Assume $\mathbb{E} m_{0}^{-\delta}<\infty$ for some $\delta>0$, (2.1) for some $\lambda>18$ and (2.2) for some $\eta>24$. Then for $t \in \mathbb{R}$, as $n \rightarrow \infty$,

$$
\begin{aligned}
\frac{1}{\Pi_{n}} Z_{n}\left(\ell_{n}+s_{n} t\right)= & \left(\Phi(t)+\frac{Q_{1, n}(t)}{n^{1 / 2}}+\frac{Q_{2, n}(t)}{n}\right) W \\
& +\left(-\frac{1}{s_{n}}\right)\left(\phi(t)+\frac{Q_{1, n}^{\prime}(t)}{n^{1 / 2}}\right) V_{1}+\frac{1}{2 !} \frac{1}{s_{n}^{2}} \phi^{\prime}(t) V_{2}+o\left(\frac{1}{n}\right) \quad \text { a.s. }
\end{aligned}
$$

where $Q_{1, n}$ is defined by (2.4) and

$$
\frac{Q_{2, n}(t)}{n}=-\frac{\left(s_{n}^{(3)}\right)^{2}}{72 s_{n}^{6}} H_{5}(t) \phi(t)-\frac{1}{24 s_{n}^{4}} \sum_{j=0}^{n-1}\left(\sigma_{j}^{(4)}-3\left(\sigma_{j}^{(2)}\right)^{2}\right) H_{3}(t) \phi(t) .
$$


Theorem 2.4 (Third order expansion). Assume $\mathbb{E} m_{0}^{-\delta}<\infty$ for some $\delta>0$, (2.1) for some $\lambda>32$ and (2.2) for some $\eta>40$. Then for $t \in \mathbb{R}$, as $n \rightarrow \infty$,

$$
\begin{aligned}
\frac{1}{\Pi_{n}} Z_{n}\left(\ell_{n}+s_{n} t\right)= & \left(\Phi(t)+\sum_{\nu=1}^{3} \frac{Q_{\nu, n}(t)}{n^{\nu / 2}}\right) W+\left(-\frac{1}{s_{n}}\right)\left(\phi(t)+\sum_{\nu=1}^{2} \frac{Q_{v, n}^{\prime}(t)}{n^{\nu / 2}}\right) V_{1} \\
& +\frac{1}{2 !} \frac{1}{s_{n}^{2}}\left(\phi^{\prime}(t)+\frac{Q_{1, n}^{\prime \prime}(t)}{n^{1 / 2}}\right) V_{2}+\frac{1}{3 !} \frac{-1}{s_{n}^{3}} \phi^{\prime \prime}(t) V_{3}+o\left(\frac{1}{n^{3 / 2}}\right) \quad \text { a.s., }
\end{aligned}
$$

where $Q_{1, n}, Q_{2, n}$ are defined by (2.4) and (2.6), and

$$
\begin{aligned}
\frac{Q_{3, n}(t)}{n^{3 / 2}}= & -\frac{\left(s_{n}^{(3)}\right)^{3}}{1296 s_{n}^{9}} H_{8}(t) \phi(t)-\frac{1}{120 s_{n}^{5}} \sum_{j=0}^{n-1}\left(\sigma_{j}^{(5)}-10 \sigma_{j}^{(3)} \sigma_{j}^{(2)}\right) H_{4}(t) \phi(t) \\
& -\frac{s_{n}^{(3)}}{144 s_{n}^{7}} \sum_{j=0}^{n-1}\left(\sigma_{j}^{(4)}-3\left(\sigma_{j}^{(2)}\right)^{2}\right) H_{6}(t) \phi(t) .
\end{aligned}
$$

The reduced versions of (2.3) and (2.5) for a branching random walk with a deterministic environment have been announced in [18].

Notice that when the branching random walk dies out, then $Z_{n}(\mathbb{R})=0$ for $n$ large enough, so that $W_{n}=N_{1, n}=N_{2, n}=N_{3, n}=0$, hence the expansions (2.3), (2.5) and (2.7) becomes trivial.

From Theorems 2.3 and 2.4, we can derive the second and third order expansions for the branching Wiener process, where the underlying branching process is a Galton-Watson process whose offspring distribution has mean $m>1$ and the motion of particles is governed by a Wiener process. For example, applying Theorem 2.4 to a constant environment and to a Gaussian moving law (for which the condition (2.2) is valid for all $\eta>0$ ), we obtain:

Corollary 2.5 (Third order expansion for the branching Wiener process). For the branching Wiener process whose offspring distribution $\left\{p_{k}: k \geq 0\right\}$ satisfies $m:=\sum_{k=0}^{\infty} k p_{k}>1$ and $\sum_{k=0}^{\infty} k(\ln k)^{1+\lambda} p_{k}<\infty$ for some $\lambda>32$, we have, for $t \in \mathbb{R}$, as $n \rightarrow \infty$,

$\frac{1}{m^{n}} Z_{n}(\sqrt{n} t)=\Phi(t) W-\frac{1}{\sqrt{n}} \phi(t) V_{1}-\frac{1}{2 n} t \phi(t) V_{2}-\frac{1}{6 n^{3 / 2}}\left(t^{2}-1\right) \phi(t) V_{3}+o\left(\frac{1}{n^{3 / 2}}\right) \quad$ a.s.

Remark 2.6. (1) This corollary extends [11], Theorem 3.2, which gave the first order expansion of the central limit theorem under the second moment condition $\sum_{k} k^{2} p_{k}<\infty$ for this model. It should be mentioned that in [36], the full expansion for the local limit theorem was obtained for the same model. However, Corollary 2.5 cannot be derived from the expansion in [36] (and vice versa). (2) A similar result can be easily formulated for the branching Wiener process in a random environment.

Inspired by Theorems 2.3 and 2.4, we have the following conjecture for the asymptotic expansion of finite order. 
Conjecture 2.7 (Finite order expansion). Let $\kappa \geq 1$ be an integer. Assume $\mathbb{E} m_{0}^{-\delta}<\infty$ for some $\delta>0,(2.1)$ and (2.2) for some $\lambda>0$ and $\eta>0$ large enough. Then

$$
\begin{aligned}
\frac{1}{\Pi_{n}} Z_{n}\left(\ell_{n}+s_{n} t\right)= & \left(\Phi(t)+\sum_{\nu=1}^{\kappa} \frac{Q_{\nu, n}(t)}{n^{\nu / 2}}\right) W \\
& +\sum_{j=1}^{\kappa} \frac{1}{j !}\left(-\frac{1}{s_{n}}\right)^{j} V_{j} \frac{d^{j}}{d t^{j}}\left(\Phi(t)+\sum_{\nu=1}^{\kappa-j} \frac{Q_{\nu, n}(t)}{n^{\nu / 2}}\right)+o\left(\frac{1}{n^{\kappa / 2}}\right) \quad \text { a.s. }
\end{aligned}
$$

where $V_{j}$ are real random variables, and

$$
\begin{aligned}
Q_{\nu, n}(x) & =\sum^{\prime}(-1)^{v+2 s} \Phi^{(v+2 s)}(x) \prod_{m=1}^{v} \frac{1}{k_{m} !}\left(\frac{\lambda_{m+2, n}}{(m+2) !}\right)^{k_{m}} \\
& =-\phi(x) \sum^{\prime} H_{\nu+2 s-1}(x) \prod_{m=1}^{v} \frac{1}{k_{m} !}\left(\frac{\lambda_{m+2, n}}{(m+2) !}\right)^{k_{m}}
\end{aligned}
$$

with the summation $\sum^{\prime}$ being carried out over all nonnegative integer solutions $\left(k_{1}, \ldots, k_{v}\right)$ of the equation $k_{1}+2 k_{2}+\cdots+v k_{v}=v, s=k_{1}+\cdots+k_{v}$ and

$$
\begin{aligned}
& \lambda_{\nu, n}=n^{(\nu-2) / 2} s_{n}^{-2 v} \sum_{j=0}^{n-1} \gamma_{\nu j}, \quad v=3,4, \ldots, k \\
& \gamma_{\nu j}=\frac{1}{i^{v}}\left[\frac{d^{\nu}}{d t^{\nu}} \log \mathbb{E}_{\xi} e^{i t\left(\widehat{L}_{j}-l_{j}\right)}\right]_{t=0}, \quad v=1,2, \ldots
\end{aligned}
$$

We remind that the term $\Phi(t)+\sum_{\nu=1}^{\kappa} n^{-v / 2} Q_{v, n}(t)$ is the Edgeworth expansion of the distribution function of sums of the random variables $\widehat{L}_{0}, \widehat{L}_{1}, \ldots$ See Lemma 3.1 below. The reader may refer to [34] for more information on the Edgeworth expansion.

For $\kappa=1,2,3$, the expansion is given respectively by (2.3), Theorem 2.3 and Theorem 2.4. By using the method proposed in this article, we should be able to prove, through tedious analysis, the expansion formula for order $\kappa=4,5$, etc. However, we have not yet found a simple and unified method valid for all $\kappa \geq 1$. This seems to need a great deal of effort and will be our future aim.

For the proofs of Theorems 2.3 and 2.4, we further develop the approaches used in [17]. Like in [17], the basic ideas are the Edgeworth expansion for an approximation of the cumulative distribution function of the sum of independent random variables (to control the position of particles in $n$th generation, which makes appear the Chebyshev-Hermite polynomials), the approximation by martingales, and a suitable decomposition of $Z_{n}(A)$ involving the independence of each particle (conditionally on the environment) from time $k_{n}=\left\lfloor n^{\beta}\right\rfloor$ for some $\beta \in(0,1)$ (see (3.2)), where $\left\lfloor n^{\beta}\right\rfloor$ denotes the integral part of $n^{\beta}$. However, the adaption of the approaches in [17] (proposed for the first order) to higher orders is far from being evident, and the progress of the 
approaches in the present article is significant. Actually, to obtain the higher order expansions, we perform much more effort than in [17]. This can be seen through three aspects. First, we need to extract more terms from the Edgeworth expansion by using Taylor's expansion, which are rather tedious due to the complexity of the Edgeworth expansion. Second, we should carefully analyze the extracted terms and suitably combine them; in particular we need to find out new martingales which appear in describing the higher order expansion, and show their convergence and their rate of convergence; furthermore, even for the known martingales $\left(N_{1, n}\right)$ and $\left(N_{2, n}\right)$, we need to investigate their convergence rates, which were not studied in the previous work [17]. Third, the time $k_{n}$ for the decomposition of the branching random walk needs to be in a smaller time range (than in [17]), to guarantee the Edgeworth expansion at a next order during the remaining time interval.

For simplicity and without any loss of generality, hereafter we will always assume that $l_{n}=0$ (otherwise, we only need to replace $L_{u i}$ by $L_{u i}-l_{n}$ ) and hence $\ell_{n}=0$. In the following, we will use $K_{\xi}$ as a constant depending on the environment, which may change from line to line.

\section{Preliminary results}

\subsection{The Edgeworth expansion for sums of independent random variables}

To begin with, we present the Edgeworth expansion for the distribution function of sums of independent random variables, which is needed to prove the main theorems. We recall the version obtained by Bai and Zhao [5], which generalizes the i.i.d. case [34], page 159, Theorem 1.

Let $\left\{X_{j}\right\}$ be independent random variables, satisfying for each $j \geq 1$

$$
\mathbb{E} X_{j}=0 \text { and } \mathbb{E}\left|X_{j}\right|^{k}<\infty
$$

for some integer $k \geq 3$. We write $B_{n}^{2}=\sum_{j=1}^{n} \mathbb{E} X_{j}^{2}$ and only consider the nontrivial case $B_{n}>0$. Let $\gamma_{v j}$ be the $\nu$-order cumulant of $X_{j}$ for each $j \geq 1$, defined by

$$
\gamma_{\nu j}=\frac{1}{i^{v}}\left[\frac{d^{\nu}}{d t^{\nu}} \log \mathbb{E} e^{i t X_{j}}\right]_{t=0}, \quad v=1,2, \ldots
$$

Write

$$
\begin{aligned}
\lambda_{\nu, n} & =n^{(v-2) / 2} B_{n}^{-v} \sum_{j=1}^{n} \gamma_{v j}, \quad v=3,4, \ldots, k ; \\
Q_{\nu, n}(x) & =\sum^{\prime}(-1)^{v+2 s} \Phi^{(v+2 s)}(x) \prod_{m=1}^{v} \frac{1}{k_{m} !}\left(\frac{\lambda_{m+2, n}}{(m+2) !}\right)^{k_{m}} \\
& =-\phi(x) \sum^{\prime} H_{\nu+2 s-1}(x) \prod_{m=1}^{v} \frac{1}{k_{m} !}\left(\frac{\lambda_{m+2, n}}{(m+2) !}\right)^{k_{m}},
\end{aligned}
$$


where the summation $\sum^{\prime}$ is carried out over all nonnegative integer solutions $\left(k_{1}, \ldots, k_{v}\right)$ of the equation $k_{1}+2 k_{2}+\cdots+v k_{v}=v$ and $s=k_{1}+\cdots+k_{v}$.

For $1 \leq j \leq n$ and $x \in \mathbb{R}$, define

$$
\begin{aligned}
F_{n}(x) & =\mathbb{P}\left(B_{n}{ }^{-1} \sum_{j=1}^{n} X_{j} \leq x\right), \quad v_{j}(t)=\mathbb{E} e^{i t X_{j}} ; \\
Y_{n j} & =X_{j} \mathbf{1}_{\left\{\left|X_{j}\right| \leq B_{n}\right\}}, \quad Z_{n j}^{(x)}=X_{j} \mathbf{1}_{\left\{\left|X_{j}\right| \leq B_{n}(1+|x|)\right\}}, \quad W_{n j}^{(x)}=X_{j} \mathbf{1}_{\left\{\left|X_{j}\right|>B_{n}(1+|x|)\right\}} .
\end{aligned}
$$

The Edgeworth expansion theorem can be stated as follows.

Lemma 3.1 ([5]). Let $n \geq 1$ and $X_{1}, \ldots, X_{n}$ be a sequence of independent random variables satisfying $B_{n}>0$. Let $k \geq 3$ be an integer such that (3.1) holds. Then

$$
\begin{aligned}
& \left|F_{n}(x)-\Phi(x)-\sum_{\nu=1}^{k-2} Q_{\nu n}(x) n^{-v / 2}\right| \\
& \leq C(k)\left\{(1+|x|)^{-k} B_{n}^{-k} \sum_{j=1}^{n} \mathbb{E}\left|W_{n j}^{(x)}\right|^{k}+(1+|x|)^{-k-1} B_{n}^{-k-1} \sum_{j=1}^{n} \mathbb{E}\left|Z_{n j}^{(x)}\right|^{k+1}\right. \\
& \left.\quad+(1+|x|)^{-k-1} n^{k(k+1) / 2}\left(\sup _{|t| \geq \delta_{n}} \frac{1}{n} \sum_{j=1}^{n}\left|v_{j}(t)\right|+\frac{1}{2 n}\right)^{n}\right\}
\end{aligned}
$$

where $\delta_{n}=\frac{1}{12} B_{n}^{2}\left(\sum_{j=1}^{n} \mathbb{E}\left|Y_{n j}\right|^{3}\right)^{-1}, C(k)>0$ is a constant depending only on $k$.

\subsection{Notation and a key decomposition}

We first introduce some notation which will be used in the sequel.

In addition to the $\sigma$-fields $\mathcal{F}_{n}$ and $\mathscr{D}_{n}$, the following $\sigma$-fields will also be used:

$$
\ell_{0}=\{\varnothing, \Omega\}, \quad \ell_{n}=\sigma\left(\xi_{k}, N_{u}, L_{u i}: k<n, i \geq 1,|u|<n\right) \quad \text { for } n \geq 1 .
$$

Define the following conditional probabilities and expectations:

$$
\mathbb{P}_{\xi, n}(\cdot)=\mathbb{P}_{\xi}\left(\cdot \mid D_{n}\right), \quad \mathbb{E}_{\xi, n}(\cdot)=\mathbb{E}_{\xi}\left(\cdot \mid D_{n}\right) ; \quad \mathbb{P}_{n}(\cdot)=\mathbb{P}\left(\cdot \mid \ell_{n}\right), \quad \mathbb{E}_{n}(\cdot)=\mathbb{E}\left(\cdot \mid \ell_{n}\right) .
$$

As usual, we write $\mathbb{N}^{*}=\{1,2,3, \ldots\}$ and denote by

$$
U=\bigcup_{n=0}^{\infty}\left(\mathbb{N}^{*}\right)^{n}
$$

the set of all finite sequences, where $\left(\mathbb{N}^{*}\right)^{0}=\{\varnothing\}$ contains the null sequence $\varnothing$. 
For all $u \in U$, let $\mathbb{T}(u)$ be the shifted tree of $\mathbb{T}$ at $u$ with defining elements $\left\{N_{u v}\right\}$ : we have (1) $\varnothing \in \mathbb{T}(u)$, (2) vi $\in \mathbb{T}(u) \Rightarrow v \in \mathbb{T}(u)$ and (3) if $v \in \mathbb{T}(u)$, then $v i \in \mathbb{T}(u)$ if and only if $1 \leq i \leq N_{u v}$. Define $\mathbb{T}_{n}(u)=\{v \in \mathbb{T}(u):|v|=n\}$. Then $\mathbb{T}=\mathbb{T}(\varnothing)$ and $\mathbb{T}_{n}=\mathbb{T}_{n}(\varnothing)$.

For $u \in\left(\mathbb{N}^{*}\right)^{k}(k \geq 0)$ and $n \geq 1$, let $S_{u}$ be the position of $u$ and write

$$
\begin{aligned}
Z_{n}(u, B) & =\sum_{v \in \mathbb{T}_{n}(u)} \mathbf{1}_{B}\left(S_{u v}-S_{u}\right), \\
Z_{n}(u, t) & =Z_{n}(u,(-\infty, t]) .
\end{aligned}
$$

Then the law of $Z_{n}(u, B)$ under $\mathbb{P}_{\xi}$ is the same as that of $Z_{n}(B)$ under $P_{\theta^{k}}$. Define

$$
\begin{aligned}
W_{n}(u, B) & =Z_{n}(u, B) / \Pi_{n}\left(\theta^{k} \xi\right), \quad W_{n}(u, t)=W_{n}(u,(-\infty, t]), \\
W_{n}(B) & =Z_{n}(B) / \Pi_{n}, \quad W_{n}(t)=W_{n}((-\infty, t]) .
\end{aligned}
$$

By definition, we have $\Pi_{n}\left(\theta^{k} \xi\right)=m_{k} \cdots m_{k+n-1}, Z_{n}(B)=Z_{n}(\varnothing, B), W_{n}(B)=W_{n}(\varnothing, B)$, $W_{n}=W_{n}(\mathbb{R})$.

For each $n$, we choose an integer $k_{n}<n$ as follows. Let $\beta \in(0,1)$ whose value will be suitably fixed in the proofs and set $k_{n}=\left\lfloor n^{\beta}\right\rfloor$, the greatest integer not bigger than $n^{\beta}$. It is apparent that

$$
Z_{n}\left(s_{n} t\right)=\sum_{u \in \mathbb{T}_{k_{n}}} Z_{n-k_{n}}\left(u, s_{n} t-S_{u}\right)
$$

from which we have the following important decomposition:

$$
\frac{1}{\Pi_{n}} Z_{n}\left(s_{n} t\right)=\mathbb{A}_{n}+\mathbb{B}_{n}
$$

with

$$
\begin{aligned}
& \mathbb{A}_{n}=\frac{1}{\Pi_{k_{n}}} \sum_{u \in \mathbb{T}_{k_{n}}}\left[W_{n-k_{n}}\left(u, s_{n} t-S_{u}\right)-\mathbb{E}_{\xi, k_{n}} W_{n-k_{n}}\left(u, s_{n} t-S_{u}\right)\right], \\
& \mathbb{B}_{n}=\frac{1}{\Pi_{k_{n}}} \sum_{u \in \mathbb{T}_{k_{n}}} \mathbb{E}_{\xi, k_{n}} W_{n-k_{n}}\left(u, s_{n} t-S_{u}\right) .
\end{aligned}
$$

\section{Proofs of Theorems 2.3 and 2.4}

\subsection{Outline of proofs}

In our proofs, we shall need the following truncations of the martingales (recall that we assume $\left.\ell_{n}=0\right)$ :

$$
\bar{W}_{k_{n}}=\frac{1}{\Pi_{k_{n}}} \sum_{u \in \mathbb{T}_{k_{n}}} \mathbf{1}_{\left\{\left|S_{u}\right| \leq k_{n}\right\}} ; \quad \bar{N}_{1, k_{n}}=\frac{1}{\Pi_{k_{n}}} \sum_{u \in \mathbb{T}_{k_{n}}} S_{u} \mathbf{1}_{\left\{\left|S_{u}\right| \leq k_{n}\right\}}
$$




$$
\begin{aligned}
& \bar{N}_{2, k_{n}}=\frac{1}{\Pi_{k_{n}}} \sum_{u \in \mathbb{T}_{k_{n}}}\left(S_{u}^{2}-s_{n}^{2}\right) \mathbf{1}_{\left\{\left|S_{u}\right| \leq k_{n}\right\}} ; \\
& \bar{N}_{3, k_{n}}=\frac{1}{\Pi_{k_{n}}} \sum_{u \in \mathbb{T}_{k_{n}}}\left(S_{u}^{3}-3 S_{u} s_{n}^{2}-s_{n}^{(3)}\right) \mathbf{1}_{\left\{\left|S_{u}\right| \leq k_{n}\right\}} .
\end{aligned}
$$

Notice that the condition $\mathbb{E} m_{0}^{-\delta}<\infty$ for some $\delta>0$ implies that $\mathbb{E}\left(\ln ^{-} m_{0}\right)^{\kappa}<\infty$ for all $\kappa>0$. Therefore Theorem 2.1 remains valid under the hypotheses of Theorems 2.3 or 2.4.

To prove Theorem 2.3, we use the decomposition (3.2) with $k_{n}=\left\lfloor n^{\beta}\right\rfloor$ and $\max \left\{\frac{3}{\lambda}, \frac{4}{\eta}\right\}<\beta<$ $\frac{1}{6}$, and we divide the proof of (2.5) into three lemmas.

Lemma 4.1. Under the hypothesis of Theorem 2.3, with $k_{n}=\left\lfloor n^{\beta}\right\rfloor$ and $\max \left\{\frac{3}{\lambda}, \frac{4}{\eta}\right\}<\beta<\frac{1}{6}$, we have

$$
n \mathbb{A}_{n} \stackrel{n \rightarrow \infty}{\longrightarrow} 0 \quad \text { a.s. }
$$

Lemma 4.2. Under the hypothesis of Theorem 2.3, with $k_{n}=\left\lfloor n^{\beta}\right\rfloor$ and $\max \left\{\frac{3}{\lambda}, \frac{4}{\eta}\right\}<\beta<\frac{1}{6}$, we have, as $n \rightarrow \infty$,

$$
\begin{aligned}
\mathbb{B}_{n}= & \left(\Phi(t)+\sum_{\nu=1}^{2} \frac{Q_{\nu, n}(t)}{n^{\nu / 2}}\right) \bar{W}_{k_{n}}+\left(-\frac{1}{s_{n}}\right)\left(\phi(t)+\frac{Q_{1, n}^{\prime}(t)}{n^{1 / 2}}\right) \bar{N}_{1, k_{n}} \\
& +\frac{1}{2 !} \frac{1}{s_{n}^{2}} \phi^{\prime}(t) \bar{N}_{2, k_{n}}+o\left(\frac{1}{n}\right) \quad \text { a.s. }
\end{aligned}
$$

Lemma 4.3. Under the hypothesis of Theorem 2.3, with $k_{n}=\left\lfloor n^{\beta}\right\rfloor$ and $\max \left\{\frac{3}{\lambda}, \frac{4}{\eta}\right\}<\beta<\frac{1}{6}$, the following assertions hold a.s. as $n \rightarrow \infty$ :

$$
\begin{aligned}
\bar{W}_{k_{n}}-W & =o\left(\frac{1}{n}\right), \\
\bar{N}_{1, k_{n}}-V_{1} & =o\left(\frac{1}{\sqrt{n}}\right), \\
\bar{N}_{2, k_{n}}-V_{2} & =o(1),
\end{aligned}
$$

where $\bar{W}_{k_{n}}, \bar{N}_{1, k_{n}}, \bar{N}_{2, k_{n}}$ are defined in (4.1), (4.2).

While in the proof of Theorem 2.4, we shall take $k_{n}=\left\lfloor n^{\beta}\right\rfloor$ with $\max \left\{\frac{4}{\lambda}, \frac{5}{\eta}\right\}<\beta<\frac{1}{8}$. We still use the decomposition (3.2), and divide the proof of (2.7) into three lemmas.

Lemma 4.4. Under the hypothesis of Theorem 2.4, with $k_{n}=\left\lfloor n^{\beta}\right\rfloor$ and $\max \left\{\frac{4}{\lambda}, \frac{5}{\eta}\right\}<\beta<\frac{1}{8}$, we have

$$
n^{3 / 2} \mathbb{A}_{n} \stackrel{n \rightarrow \infty}{\longrightarrow} 0 \quad \text { a.s. }
$$


Lemma 4.5. Under the hypothesis of Theorem 2.4, with $k_{n}=\left\lfloor n^{\beta}\right\rfloor$ and $\max \left\{\frac{4}{\lambda}, \frac{5}{\eta}\right\}<\beta<\frac{1}{8}$, the following holds a.s. as $n \rightarrow \infty$ :

$$
\begin{aligned}
\mathbb{B}_{n}= & \left(\Phi(t)+\sum_{\nu=1}^{3} \frac{Q_{\nu, n}(t)}{n^{\nu / 2}}\right) \bar{W}_{k_{n}}+\left(-\frac{1}{s_{n}}\right)\left(\phi(t)+\sum_{\nu=1}^{2} \frac{Q_{\nu, n}^{\prime}(t)}{n^{\nu / 2}}\right) \bar{N}_{1, k_{n}} \\
& +\frac{1}{2 !} \frac{1}{s_{n}^{2}}\left(\phi^{\prime}(t)+\frac{Q_{1, n}^{\prime \prime}(t)}{n^{1 / 2}}\right) \bar{N}_{2, k_{n}}+\frac{1}{3 !}\left(-\frac{1}{s_{n}^{3}}\right) \phi^{\prime \prime}(t) \bar{N}_{3, k_{n}}+o\left(\frac{1}{n^{3 / 2}}\right) .
\end{aligned}
$$

Lemma 4.6. Under the hypothesis of Theorem 2.4 , with $k_{n}=\left\lfloor n^{\beta}\right\rfloor$ and $\max \left\{\frac{4}{\lambda}, \frac{5}{\eta}\right\}<\beta<\frac{1}{8}$, the following assertions hold a.s. as $n \rightarrow \infty$ :

$$
\begin{aligned}
\bar{W}_{k_{n}}-W & =o\left(\frac{1}{n^{3 / 2}}\right), \\
\bar{N}_{1, k_{n}}-V_{1} & =o\left(\frac{1}{n}\right), \\
\bar{N}_{2, k_{n}}-V_{2} & =o\left(\frac{1}{\sqrt{n}}\right), \\
\bar{N}_{3, k_{n}}-V_{3} & =o(1),
\end{aligned}
$$

where $\bar{W}_{k_{n}}, \bar{N}_{1, k_{n}}, \bar{N}_{2, k_{n}}, \bar{N}_{3, k_{n}}$ are defined in (4.1), (4.2), (4.3).

To avoid repetition, here we shall only present the proofs of Lemmas 4.4, 4.5 and 4.6; similar arguments apply to Lemmas 4.1, 4.2 and 4.3.

\subsection{Proofs of Lemmas 4.4, 4.5 and 4.6}

Proof of Lemma 4.4. The proof is similar to that of Lemma 5.1 in [17]. For ease of notation, we will denote by $[f(x)]_{x=a}$ the value of a function $f(x)$ at the point $a$, and define for $|u|=k_{n}$,

$$
\begin{aligned}
X_{n, u} & =W_{n-k_{n}}\left(u, s_{n} t-S_{u}\right)-\mathbb{E}_{\xi, k_{n}} W_{n-k_{n}}\left(u, s_{n} t-S_{u}\right), \quad \bar{X}_{n, u}=X_{n, u} \mathbf{1}_{\left\{\left|X_{n, u}\right|<\Pi_{k_{n}}\right\}}, \\
\overline{\mathbb{A}}_{n} & =\frac{1}{\Pi_{k_{n}}} \sum_{u \in \mathbb{T}_{k_{n}}} \bar{X}_{n, u} .
\end{aligned}
$$

Then we see that $\left|X_{n, u}\right| \leq W_{n-k_{n}}(u)+1$.

To prove Lemma 4.4, we will use the extended Borel-Cantelli lemma. We can obtain the required result once we prove that $\forall \varepsilon>0$,

$$
\sum_{n=1}^{\infty} \mathbb{P}_{k_{n}}\left(\left|n^{3 / 2} \mathbb{A}_{n}\right|>2 \varepsilon\right)<\infty
$$


Notice that

$$
\begin{aligned}
& \mathbb{P}_{k_{n}}\left(\left|\mathbb{A}_{n}\right|>\frac{2 \varepsilon}{n^{3 / 2}}\right) \\
& \quad \leq \mathbb{P}_{k_{n}}\left(\mathbb{A}_{n} \neq \overline{\mathbb{A}}_{n}\right)+\mathbb{P}_{k_{n}}\left(\left|\overline{\mathbb{A}}_{n}-\mathbb{E}_{\xi, k_{n}} \overline{\mathbb{A}}_{n}\right|>\frac{\varepsilon}{n^{3 / 2}}\right)+\mathbb{P}_{k_{n}}\left(\left|\mathbb{E}_{\xi, k_{n}} \overline{\mathbb{A}}_{n}\right|>\frac{\varepsilon}{n^{3 / 2}}\right) .
\end{aligned}
$$

Then we can proceed the proof in 3 steps.

Step 1 . We first prove that

$$
\sum_{n=1}^{\infty} \mathbb{P}_{k_{n}}\left(\mathbb{A}_{n} \neq \overline{\mathbb{A}}_{n}\right)<\infty
$$

To this end, define

$$
W^{*}=\sup _{n} W_{n}
$$

We need the following result on $W^{*}$.

Lemma 4.7 ([31], Theorem 1.2). Assume (2.1) for some $\lambda>0$ and $\mathbb{E} m_{0}^{-\delta}<\infty$ for some $\delta>0$. Then

$$
\mathbb{E}\left(W^{*}+1\right)\left(\ln \left(W^{*}+1\right)\right)^{\lambda}<\infty
$$

Observe that

$$
\begin{aligned}
\mathbb{P}_{k_{n}}\left(\mathbb{A}_{n} \neq \overline{\mathbb{A}}_{n}\right) & \leq \sum_{u \in \mathbb{T}_{k_{n}}} \mathbb{P}_{k_{n}}\left(X_{n, u} \neq \bar{X}_{n, u}\right)=\sum_{u \in \mathbb{T}_{k_{n}}} \mathbb{P}_{k_{n}}\left(\left|X_{n, u}\right| \geq \Pi_{k_{n}}\right) \\
& \leq \sum_{u \in \mathbb{T}_{k_{n}}} \mathbb{P}_{k_{n}}\left(W_{n-k_{n}}(u)+1 \geq \Pi_{k_{n}}\right) \\
& =W_{k_{n}}\left[r_{n} \mathbb{P}\left(W_{n-k_{n}}+1 \geq r_{n}\right)\right]_{r_{n}=\Pi_{k_{n}}} \\
& \leq W_{k_{n}}\left[\mathbb{E}\left(\left(W_{n-k_{n}}+1\right) \mathbf{1}_{\left\{W_{n-k_{n}}+1 \geq r_{n}\right\}}\right)\right]_{r_{n}=\Pi_{k_{n}}} \\
& \leq W_{k_{n}}\left[\mathbb{E}\left(\left(W^{*}+1\right) \mathbf{1}_{\left\{W^{*}+1 \geq r_{n}\right\}}\right)\right]_{r_{n}=\Pi_{k_{n}}} \\
& \leq W^{*}\left(\ln \Pi_{k_{n}}\right)^{-\lambda} \mathbb{E}\left(W^{*}+1\right)\left(\ln \left(W^{*}+1\right)\right)^{\lambda} \\
& \leq K_{\xi} W^{*} n^{-\lambda \beta} \mathbb{E}\left(W^{*}+1\right)\left(\ln \left(W^{*}+1\right)\right)^{\lambda}
\end{aligned}
$$

where the last inequality holds since

$$
\frac{1}{n} \ln \Pi_{n} \rightarrow \mathbb{E} \ln m_{0}>0 \quad \text { a.s. }
$$


and $k_{n} \sim n^{\beta}$. By the choice of $\beta$ and Lemma 4.7, we obtain (4.16).

Step 2. We next prove that $\forall \varepsilon>0$,

$$
\sum_{n=1}^{\infty} \mathbb{P}_{k_{n}}\left(\left|\overline{\mathbb{A}}_{n}-\mathbb{E}_{\xi, k_{n}} \overline{\mathbb{A}}_{n}\right|>\frac{\varepsilon}{n^{3 / 2}}\right)<\infty
$$

Take a constant $b \in\left(1, e^{\mathbb{E} \ln m_{0}}\right)$. Observe that $\forall u \in \mathbb{T}_{k_{n}}, n \geq 1$,

$$
\begin{aligned}
\mathbb{E}_{k_{n}} \bar{X}_{n, u}^{2} & =\int_{0}^{\infty} 2 x \mathbb{P}_{k_{n}}\left(\left|\bar{X}_{n, u}\right|>x\right) d x=2 \int_{0}^{\infty} x \mathbb{P}_{k_{n}}\left(\left|X_{n, u}\right| \mathbf{1}_{\left\{\left|X_{n, u}\right|<\Pi_{k_{n}}\right\}}>x\right) d x \\
& \leq 2 \int_{0}^{\Pi_{k_{n}}} x \mathbb{P}_{k_{n}}\left(\left|W_{n-k_{n}}(u)+1\right|>x\right) d x=2 \int_{0}^{\Pi_{k_{n}}} x \mathbb{P}\left(\left|W_{n-k_{n}}+1\right|>x\right) d x \\
& \leq 2 \int_{0}^{\Pi_{k_{n}}} x \mathbb{P}\left(W^{*}+1>x\right) d x \\
& \leq 2 \int_{e}^{\Pi_{k_{n}}}(\ln x)^{-\lambda} \mathbb{E}\left(W^{*}+1\right)\left(\ln \left(W^{*}+1\right)\right)^{\lambda} d x+2 \int_{0}^{e} x d x \\
& \leq 2 \mathbb{E}\left(W^{*}+1\right)\left(\ln \left(W^{*}+1\right)\right)^{\lambda}\left(\int_{e}^{b^{k_{n}}}(\ln x)^{-\lambda} d x+\int_{b^{k_{n}}}^{\Pi_{k_{n}}}(\ln x)^{-\lambda} d x\right)+e^{2} \\
& \leq 2 \mathbb{E}\left(W^{*}+1\right)\left(\ln \left(W^{*}+1\right)\right)^{\lambda}\left(b^{k_{n}}+\left(\Pi_{k_{n}}-b^{k_{n}}\right)\left(k_{n} \ln b\right)^{-\lambda}\right)+e^{2} .
\end{aligned}
$$

Then we have that

$$
\begin{aligned}
& \sum_{n=1}^{\infty} \mathbb{P}_{k_{n}}\left(\left|\overline{\mathbb{A}}_{n}-\mathbb{E}_{\xi, k_{n}} \overline{\mathbb{A}}_{n}\right|>\frac{\varepsilon}{n^{3 / 2}}\right) \\
& =\sum_{n=1}^{\infty} \mathbb{E}_{k_{n}} \mathbb{P}_{\xi, k_{n}}\left(\left|\overline{\mathbb{A}}_{n}-\mathbb{E}_{\xi, k_{n}} \overline{\mathbb{A}}_{n}\right|>\frac{\varepsilon}{n^{3 / 2}}\right) \\
& \leq \varepsilon^{-2} \sum_{n=1}^{\infty} n^{3} \mathbb{E}_{k_{n}}\left(\Pi_{k_{n}}^{-2} \sum_{u \in \mathbb{T}_{k_{n}}} \mathbb{E}_{\xi, k_{n}} \bar{X}_{n, u}^{2}\right)=\varepsilon^{-2} \sum_{n=1}^{\infty} n^{3}\left(\Pi_{k_{n}}^{-2} \sum_{u \in \mathbb{T}_{k_{n}}} \mathbb{E}_{k_{n}} \bar{X}_{n, u}^{2}\right) \\
& \leq \varepsilon^{-2} \sum_{n=1}^{\infty} \frac{n^{3} W_{k_{n}}}{\Pi_{k_{n}}}\left[2 \mathbb{E}\left(W^{*}+1\right)\left(\ln \left(W^{*}+1\right)^{\lambda}\right)\left(b^{k_{n}}+\left(\Pi_{k_{n}}-b^{k_{n}}\right)\left(k_{n} \ln b\right)^{-\lambda}\right)+e^{2}\right] \\
& \leq 2 \varepsilon^{-2} W^{*} \mathbb{E}\left(W^{*}+1\right)\left(\ln \left(W^{*}+1\right)^{\lambda}\right)\left(\sum_{n=1}^{\infty} \frac{n^{3}}{\Pi_{k_{n}}} b^{k_{n}}+\sum_{n=1}^{\infty} n^{3}\left(k_{n} \ln b\right)^{-\lambda}\right) \\
& +e^{2} \varepsilon^{-2} W^{*} \sum_{n=1}^{\infty} \frac{n^{3}}{\Pi_{k_{n}}} .
\end{aligned}
$$


By (4.18) and $\lambda \beta>4$, the three series in the last expression above converge under our hypothesis and hence (4.19) is proved.

Step 3. Observe

$$
\begin{aligned}
& \mathbb{P}_{k_{n}}\left(\left|\mathbb{E}_{\xi, k_{n}} \overline{\mathbb{A}}_{n}\right|>\frac{\varepsilon}{n^{3 / 2}}\right) \\
& \leq \frac{n^{3 / 2}}{\varepsilon} \mathbb{E}_{k_{n}}\left|\mathbb{E}_{\xi, k_{n}} \overline{\mathbb{A}}_{n}\right|=\frac{n^{3 / 2}}{\varepsilon} \mathbb{E}_{k_{n}}\left|\frac{1}{\Pi_{k_{n}}} \sum_{u \in \mathbb{T}_{k_{n}}} \mathbb{E}_{\xi, k_{n}} \bar{X}_{n, u}\right| \\
& =\frac{n^{3 / 2}}{\varepsilon} \mathbb{E}_{k_{n}}\left|\frac{1}{\Pi_{k_{n}}} \sum_{u \in \mathbb{T}_{k_{n}}}\left(-\mathbb{E}_{\xi, k_{n}} X_{n, u} \mathbf{1}_{\left\{\left|X_{n, u}\right| \geq \Pi_{k_{n}}\right\}}\right)\right| \\
& \leq \frac{n^{3 / 2}}{\varepsilon} \frac{1}{\Pi_{k_{n}}} \sum_{u \in \mathbb{T}_{k_{n}}} \mathbb{E}_{k_{n}}\left(W_{n-k_{n}}(u)+1\right) \mathbf{1}_{\left\{W_{n-k_{n}}(u)+1 \geq \Pi_{k_{n}}\right\}} \\
& =\frac{n^{3 / 2} W_{k_{n}}}{\varepsilon}\left[\mathbb{E}\left(W_{n-k_{n}}+1\right) \mathbf{1}_{\left\{W_{n-k_{n}}+1 \geq r_{n}\right\}}\right]_{r_{n}=\Pi_{k_{n}}} \\
& \leq \frac{W^{*}}{\varepsilon} n^{3 / 2}\left[\mathbb{E}\left(W^{*}+1\right) \mathbf{1}_{\left\{W^{*}+1 \geq r_{n}\right\}}\right]_{r_{n}=\Pi_{k_{n}}} \\
& \leq \frac{W^{*}}{\varepsilon} \frac{n^{3 / 2}}{\left(\ln \Pi_{k_{n}}\right)^{\lambda}} \mathbb{E}\left(W^{*}+1\right) \ln ^{\lambda}\left(W^{*}+1\right) \\
& \leq \frac{W^{*}}{\varepsilon} K_{\xi} n^{3 / 2-\lambda \beta} \mathbb{E}\left(W^{*}+1\right) \ln ^{\lambda}\left(W^{*}+1\right) .
\end{aligned}
$$

Then by (4.18) and $\lambda \beta>4$, it follows that

$$
\sum_{n=1}^{\infty} \mathbb{P}_{k_{n}}\left(\left|\mathbb{E}_{\xi, k_{n}} \overline{\mathbb{A}}_{n}\right|>\frac{\varepsilon}{n^{3 / 2}}\right)<\infty
$$

Combining Steps 1-3, we obtain (4.15). Hence the lemma is proved.

Proof of Lemma 4.5. For ease of reference, we introduce some notation:

$$
\begin{array}{ll}
\kappa_{1, n}=\frac{1}{6}\left(s_{n}^{2}-s_{k_{n}}^{2}\right)^{-3 / 2}\left(s_{n}^{(3)}-s_{k_{n}}^{(3)}\right), & D_{1}(x)=-H_{2}(x) \phi(x), \\
\kappa_{2, n}=\frac{1}{72}\left(s_{n}^{2}-s_{k_{n}}^{2}\right)^{-3}\left(s_{n}^{(3)}-s_{k_{n}}^{(3)}\right)^{2}, & D_{2}(x)=-H_{5}(x) \phi(x), \\
\kappa_{3, n}=\frac{1}{24}\left(s_{n}^{2}-s_{k_{n}}^{2}\right)^{-2} \sum_{j=k_{n}}^{n-1}\left(\sigma_{j}^{(4)}-3\left(\sigma_{j}^{(2)}\right)^{2}\right), & D_{3}(x)=-H_{3}(x) \phi(x),
\end{array}
$$




$$
\begin{aligned}
R_{n}(x)= & -\frac{\left(s_{n}^{(3)}-s_{k_{n}}^{(3)}\right)^{3}}{1296\left(s_{n}^{2}-s_{k_{n}}^{2}\right)^{9 / 2}} H_{8}(x) \phi(x)-\frac{\sum_{j=k_{n}}^{n-1}\left(\sigma_{j}^{(5)}-10 \sigma_{j}^{(3)} \sigma_{j}^{(2)}\right)}{120\left(s_{n}^{2}-s_{k_{n}}^{2}\right)^{5 / 2}} H_{4}(x) \phi(x) \\
& -\frac{\left(s_{n}^{(3)}-s_{k_{n}}^{(3)}\right) \sum_{j=k_{n}}^{n-1}\left(\sigma_{j}^{(4)}-3\left(\sigma_{j}^{(2)}\right)^{2}\right)}{144\left(s_{n}^{2}-s_{k_{n}}^{2}\right)^{7 / 2}} H_{6}(x) \phi(x) .
\end{aligned}
$$

Observe that

$$
\mathbb{B}_{n}=\mathbb{B}_{n 1}+\mathbb{B}_{n 2}+\mathbb{B}_{n 3}
$$

where

$$
\begin{aligned}
\mathbb{B}_{n 1}= & \frac{1}{\Pi_{k_{n}}} \sum_{u \in \mathbb{T}_{k_{n}}} \mathbf{1}_{\left\{\left|S_{u}\right|>k_{n}\right\}}\left[\mathbb{E}_{\xi, k_{n}} W_{n-k_{n}}\left(u, s_{n} t-S_{u}\right)\right], \\
\mathbb{B}_{n 2}= & \frac{1}{\Pi_{k_{n}}} \sum_{u \in \mathbb{T}_{k_{n}}} \mathbf{1}_{\left\{\left|S_{u}\right| \leq k_{n}\right\}}\left[\mathbb{E}_{\xi, k_{n}} W_{n-k_{n}}\left(u, s_{n} t-S_{u}\right)-\Phi\left(\frac{s_{n} t-S_{u}}{\left(s_{n}^{2}-s_{k_{n}}^{2}\right)^{1 / 2}}\right)\right. \\
& \left.-\sum_{\nu=1}^{3} \kappa_{\nu, n} D_{\nu}\left(\frac{s_{n} t-S_{u}}{\left(s_{n}^{2}-s_{k_{n}}^{2}\right)^{1 / 2}}\right)-R_{n}\left(\frac{s_{n} t-S_{u}}{\left(s_{n}^{2}-s_{k_{n}}^{2}\right)^{1 / 2}}\right)\right], \\
\mathbb{B}_{n 3}= & \frac{1}{\Pi_{k_{n}}} \sum_{u \in \mathbb{T}_{k_{n}}} \mathbf{1}_{\left\{\left|S_{u}\right| \leq k_{n}\right\}}\left[\Phi\left(\frac{s_{n} t-S_{u}}{\left(s_{n}^{2}-s_{k_{n}}^{2}\right)^{1 / 2}}\right)+\sum_{\nu=1}^{3} \kappa_{\nu, n} D_{\nu}\left(\frac{s_{n} t-S_{u}}{\left(s_{n}^{2}-s_{k_{n}}^{2}\right)^{1 / 2}}\right)\right. \\
& \left.+R_{n}\left(\frac{s_{n} t-S_{u}}{\left(s_{n}^{2}-s_{k_{n}}^{2}\right)^{1 / 2}}\right)\right] .
\end{aligned}
$$

The lemma will be proved once we show that a.s.

$$
\begin{aligned}
& n^{3 / 2} \mathbb{B}_{n 1} \stackrel{n \rightarrow \infty}{\longrightarrow} 0, \\
& n^{3 / 2} \mathbb{B}_{n 2} \stackrel{n \rightarrow \infty}{\longrightarrow} 0, \\
& \mathbb{B}_{n 3}=\left(\Phi(t)+\sum_{\nu=1}^{3} \frac{Q_{\nu, n}(t)}{n^{\nu / 2}}\right) \bar{W}_{k_{n}}+\left(-\frac{1}{s_{n}}\right)\left(\phi(t)+\sum_{\nu=1}^{2} \frac{Q_{\nu, n}^{\prime}(t)}{n^{\nu / 2}}\right) \bar{N}_{1, k_{n}} \\
& \quad+\frac{1}{2 !} \frac{1}{s_{n}^{2}}\left(\phi^{\prime}(t)+\frac{Q_{1, n}^{\prime \prime}(t)}{n^{1 / 2}}\right) \bar{N}_{2, k_{n}}+\frac{1}{3 !}\left(-\frac{1}{s_{n}^{3}}\right) \phi^{\prime \prime}(t) \bar{N}_{3, k_{n}}+o\left(\frac{1}{n^{3 / 2}}\right),
\end{aligned}
$$

where $\bar{W}_{k_{n}}, \bar{N}_{1, k_{n}}, \bar{N}_{2, k_{n}}, \bar{N}_{3, k_{n}}$ are defined in (4.1)-(4.3). We will prove these results subsequently. 
First, we prove (4.21). Since

$$
\left|\mathbb{B}_{n 1}\right| \leq \frac{1}{\prod_{k_{n}}} \sum_{u \in \mathbb{T}_{k_{n}}} \mathbf{1}_{\left\{\left|S_{u}\right|>k_{n}\right\}},
$$

it will follow from the following fact:

$$
n^{3 / 2} \frac{1}{\prod_{k_{n}}} \sum_{u \in \mathbb{T}_{k_{n}}} \mathbf{1}_{\left\{\left|S_{u}\right|>k_{n}\right\}} \stackrel{n \rightarrow \infty}{\longrightarrow} 0 \quad \text { a.s. }
$$

In order to prove (4.24), we first observe that

$$
\begin{aligned}
\mathbb{E}\left(\sum_{n=1}^{\infty} n^{3 / 2} \frac{1}{\Pi_{k_{n}}} \sum_{u \in \mathbb{T}_{k_{n}}} \mathbf{1}_{\left\{\left|S_{u}\right|>k_{n}\right\}}\right) & =\sum_{n=1}^{\infty} n^{3 / 2} \mathbb{E} \mathbf{1}_{\left\{\left|\widehat{S}_{k_{n}}\right|>k_{n}\right\}} \leq \sum_{n=1}^{\infty} n^{3 / 2} k_{n}^{-\eta} \mathbb{E}\left|\widehat{S}_{k_{n}}\right|^{\eta} \\
& \leq \sum_{n=1}^{\infty} n^{3 / 2} k_{n}^{-\frac{\eta}{2}-1} \sum_{j=0}^{k_{n}-1} \mathbb{E}\left|\widehat{L}_{j}\right|^{\eta}=\sum_{n=1}^{\infty} n^{3 / 2} k_{n}^{-\frac{\eta}{2}} \mathbb{E}\left|\widehat{L}_{0}\right|^{\eta}
\end{aligned}
$$

where $\widehat{S}_{k_{n}}=\sum_{j=0}^{k_{n}-1} \widehat{L}_{j}$. By the choice of $\beta$ and $k_{n}, 3 / 2-\beta \eta / 2<-1$ and the series in the right-hand side of the above expression converges. So

$$
\sum_{n=1}^{\infty} n^{3 / 2} \frac{1}{\Pi_{k_{n}}} \sum_{u \in \mathbb{T}_{k_{n}}} \mathbf{1}_{\left\{\left|S_{u}\right|>k_{n}\right\}}<\infty \quad \text { a.s. }
$$

which implies (4.24), and consequently (4.21) follows.

The proof of (4.22) will mainly be based on the following result about the asymptotic expansion of the distribution of the sum of random variables.

Proposition 4.8. Under the hypothesis of Theorem 2.4, for a.e. $\xi$,

$$
\varepsilon_{n}:=n^{\frac{3}{2}} \sup _{x \in \mathbb{R}}\left|\mathbb{P}_{\xi}\left(\frac{\sum_{k=k_{n}}^{n-1} \widehat{L}_{k}}{\left(s_{n}^{2}-s_{k_{n}}^{2}\right)^{1 / 2}} \leq x\right)-\Phi(x)-\sum_{\nu=1}^{3} \kappa_{\nu, n} D_{\nu}(x)-R_{n}(x)\right| \stackrel{n \rightarrow \infty}{\longrightarrow} 0 .
$$

Proof. Denote by $v_{k}=v\left(\xi_{k}\right)$ the characteristic function of the random distribution $G\left(\xi_{k}\right)$, which is also the characteristic function of $\widehat{L}_{k}$ under $\mathbb{P}_{\xi}$ : for all real $t, v_{k}(t)=\int e^{i t x} G\left(\xi_{k}\right)(d x)=$ $\mathbb{E}_{\xi} e^{i t \widehat{L}_{k}}$. Combining the Markov inequality with Lemma 3.1, we obtain the following result:

$$
\begin{aligned}
& \sup _{x \in \mathbb{R}}\left|\mathbb{P}_{\xi}\left(\frac{\sum_{k=k_{n}}^{n-1} \widehat{L}_{k}}{\left(s_{n}^{2}-s_{k_{n}}^{2}\right)^{1 / 2}} \leq x\right)-\Phi(x)-\sum_{\nu=1}^{3} \kappa_{\nu, n} D_{\nu}(x)-R_{n}(x)\right| \\
& \quad \leq K_{\xi}\left\{\left(s_{n}^{2}-s_{k_{n}}^{2}\right)^{-3} \sum_{j=k_{n}}^{n-1} \mathbb{E}_{\xi}\left|\widehat{L}_{j}\right|^{6}+n^{15}\left(\sup _{|t|>T} \frac{1}{n}\left(k_{n}+\sum_{j=k_{n}}^{n-1}\left|v_{j}(t)\right|\right)+\frac{1}{2 n}\right)^{n}\right\} .
\end{aligned}
$$


By our conditions on the environment, we know that

$$
\lim _{n \rightarrow \infty} n^{2}\left(s_{n}^{2}-s_{k_{n}}^{2}\right)^{-3} \sum_{j=k_{n}}^{n-1} \mathbb{E}_{\xi}\left|\widehat{L}_{k}\right|^{6}=\mathbb{E}\left|\widehat{L}_{0}\right|^{6} /\left(\mathbb{E} \sigma_{0}^{(2)}\right)^{3} .
$$

By (2.2), $v_{0}$ satisfies

$$
\mathbb{P}\left(\limsup _{|t| \rightarrow \infty}\left|v_{0}(t)\right|<1\right)>0 .
$$

So there exist constants $T>0$ and $0<c<1$ such that $\mathbb{P}\left(\sup _{|t|>T}\left|v_{0}(t)\right|<c\right)>0$. Since $v_{n}$ has the same law as $v_{0}$, it follows that $\mathbb{P}\left(\sup _{|t|>T}\left|v_{n}(t)\right|<c\right)>0$. Define $c\left(\xi_{n}\right)=c$ if the characteristic function $v_{n}=v\left(\xi_{n}\right)$ of $G\left(\xi_{n}\right)$ satisfies $\sup _{|t|>T}\left|v_{n}(t)\right|<c$, and $c\left(\xi_{n}\right)=1$ otherwise. Then $c_{n}:=c\left(\xi_{n}\right)$ satisfies $0<c_{n} \leq 1$ (in fact $c_{n}=c$ or 1 ),

$$
\sup _{|t|>T}\left|v_{n}(t)\right| \leq c_{n} \quad \text { and } \quad \mathbb{P}\left(c_{n}<1\right)>0 .
$$

Consequently, by the law of large numbers, we have

$$
\sup _{|t|>T}\left(\frac{1}{n} \sum_{j=k_{n}}^{n-1}\left|v_{j}(t)\right|\right) \leq \frac{1}{n} \sum_{j=1}^{n-1} c_{j} \rightarrow \mathbb{E} c_{0}<1 .
$$

Then for $n$ large enough,

$$
\left(\sup _{|t|>T} \frac{1}{n}\left(k_{n}+\sum_{j=k_{n}}^{n-1}\left|v_{j}(t)\right|\right)+\frac{1}{2 n}\right)^{n}=o\left(n^{-m}\right), \quad \forall m>0 .
$$

The proposition comes from (4.25) and (4.26).

Observe that for $u \in \mathbb{T}_{k_{n}}$,

$$
\mathbb{E}_{\xi, k_{n}} W_{n-k_{n}}\left(u, s_{n} t-S_{u}\right)=\left.\mathbb{P}_{\xi}\left(\frac{\sum_{k=k_{n}}^{n-1} \widehat{L}_{k}}{\left(s_{n}^{2}-s_{k_{n}}^{2}\right)^{1 / 2}} \leq x\right)\right|_{x=s_{n} t-S_{u}} .
$$

From Proposition 4.8, it follows that

$$
n^{3 / 2}\left|\mathbb{B}_{n 2}\right| \leq W_{k_{n}} \varepsilon_{n} \stackrel{n \rightarrow \infty}{\longrightarrow} 0 .
$$

Hence (4.22) is proved.

It remains to prove (4.23). Our arguments will depend heavily on Taylor's expansion with tedious calculus. In the following, we shall use the notation $\varepsilon_{n}^{*}$ to denote an infinitesimal (which may change from line to line) dominated by another one $a_{n}$ depending only on the environment $\xi$ and on the value of $t$ : that is

$$
\left|\varepsilon_{n}^{*}\right| \leq a_{n}=a_{n}(\xi, t) \longrightarrow 0 \quad \text { as } n \rightarrow \infty .
$$


Below we suppose always that $u \in \mathbb{T}_{k_{n}}$ and $\left|S_{u}\right| \leq k_{n}$. Then

$$
\begin{aligned}
\frac{s_{n} t-S_{u}}{\sqrt{s_{n}^{2}-s_{k_{n}}^{2}}}-t & =\left[\left(1-\frac{s_{k_{n}}^{2}}{s_{n}^{2}}\right)^{-1 / 2}-1\right] t-\left(1-\frac{s_{k_{n}}^{2}}{s_{n}^{2}}\right)^{-1 / 2} \frac{S_{u}}{s_{n}} \\
& =\left[1+\frac{s_{k_{n}}^{2}}{2 s_{n}^{2}}+\varepsilon_{n}^{*} n^{-3 / 2}-1\right] t-\left[1+\frac{s_{k_{n}}^{2}}{2 s_{n}^{2}}+\varepsilon_{n}^{*} n^{-3 / 2}\right] \frac{S_{u}}{s_{n}} \\
& =-\frac{S_{u}}{s_{n}}+\frac{s_{k_{n}}^{2} t}{2 s_{n}^{2}}-\frac{s_{k_{n}}^{2} S_{u}}{2 s_{n}^{3}}+\varepsilon_{n}^{*} n^{-3 / 2}
\end{aligned}
$$

Further, it is easy to see that

$$
\begin{aligned}
& \left(\frac{s_{n} t-S_{u}}{\sqrt{s_{n}^{2}-s_{k_{n}}^{2}}}-t\right)^{2}=\frac{S_{u}^{2}}{s_{n}^{2}}-\frac{s_{k_{n}}^{2} S_{u} t}{s_{n}^{3}}+\varepsilon_{n}^{*} n^{-3 / 2} \\
& \left(\frac{s_{n} t-S_{u}}{\sqrt{s_{n}^{2}-s_{k_{n}}^{2}}}-t\right)^{3}=-\frac{S_{u}^{3}}{s_{n}^{3}}+\varepsilon_{n}^{*} n^{-3 / 2} .
\end{aligned}
$$

By Taylor's expansion and the above estimates,

$$
\begin{aligned}
& \Phi\left(\frac{s_{n} t-S_{u}}{\left(s_{n}^{2}-s_{k_{n}}^{2}\right)^{1 / 2}}\right) \\
& =\Phi(t)+\sum_{j=1}^{3} \frac{1}{j !} \Phi^{(j)}(t)\left(\frac{s_{n} t-S_{u}}{\sqrt{s_{n}^{2}-s_{k_{n}}^{2}}}-t\right)^{j}+\varepsilon_{n}^{*} n^{-3 / 2} \\
& =\Phi(t)-\frac{1}{s_{n}} \phi(t) S_{u}-\frac{1}{2 s_{n}^{2}} t \phi(t)\left(S_{u}^{2}-s_{k_{n}}^{2}\right)-\frac{1}{6 s_{n}^{3}} \phi(t) H_{2}(t)\left(S_{u}^{3}-3 s_{k_{n}}^{2} S_{u}\right) \\
& \quad+\varepsilon_{n}^{*} n^{-3 / 2} .
\end{aligned}
$$

Since

$$
\begin{aligned}
\kappa_{1, n} & =\frac{1}{6 s_{n}^{3}}\left(1-\frac{s_{k_{n}}^{2}}{s_{n}^{2}}\right)^{-3 / 2}\left(s_{n}^{(3)}-s_{k_{n}}^{(3)}\right) \\
& =\frac{1}{6 s_{n}^{3}}\left(1+\frac{3 s_{k_{n}}^{2}}{2 s_{n}^{2}}+\varepsilon_{n}^{*} n^{-1}\right)\left(s_{n}^{(3)}-s_{k_{n}}^{(3)}\right) \\
& =\frac{1}{6 s_{n}^{3}} s_{n}^{(3)}-\frac{1}{6 s_{n}^{3}} s_{k_{n}}^{(3)}+\frac{s_{n}^{(3)} s_{k_{n}}^{2}}{4 s_{n}^{5}}+\varepsilon_{n}^{*} n^{-3 / 2}
\end{aligned}
$$


and

$$
\begin{aligned}
& D_{1}\left(\frac{s_{n} t-S_{u}}{\left(s_{n}^{2}-s_{k_{n}}^{2}\right)^{1 / 2}}\right) \\
& \quad=D_{1}(t)+D_{1}^{\prime}(t)\left(\frac{s_{n} t-S_{u}}{\sqrt{s_{n}^{2}-s_{k_{n}}^{2}}}-t\right)+\frac{1}{2} D_{1}^{\prime \prime}(t)\left(\frac{s_{n} t-S_{u}}{\sqrt{s_{n}^{2}-s_{k_{n}}^{2}}}-t\right)^{2}+\varepsilon_{n}^{*} n^{-1} \\
& \quad=-H_{2}(t) \phi(t)+H_{3}(t) \phi(t)\left(-\frac{S_{u}}{s_{n}}+\frac{s_{k_{n}}^{2} t}{2 s_{n}^{2}}\right)-\frac{1}{2 s_{n}^{2}} H_{4}(t) \phi(t) S_{u}^{2}+\varepsilon_{n}^{*} n^{-1}
\end{aligned}
$$

we obtain

$$
\begin{aligned}
\kappa_{1, n} D_{1} & \left(\frac{s_{n} t-S_{u}}{\sqrt{s_{n}^{2}-s_{k_{n}}^{2}}}\right) \\
= & -\frac{1}{6 s_{n}^{3}} s_{n}^{(3)} H_{2}(t) \phi(t)+\frac{1}{6 s_{n}^{3}} s_{k_{n}}^{(3)} H_{2}(t) \phi(t)-\frac{1}{6 s_{n}^{4}} s_{n}^{(3)} S_{u} H_{3}(t) \phi(t) \\
& -\frac{s_{n}^{(3)} s_{k_{n}}^{2}}{4 s_{n}^{5}} H_{2}(t) \phi(t)+\frac{1}{12 s_{n}^{5}} s_{n}^{(3)} s_{k_{n}}^{2} t H_{3}(t) \phi(t) \\
& -\frac{1}{12 s_{n}^{5}} s_{n}^{(3)} S_{u}^{2} H_{4}(t) \phi(t)+\varepsilon_{n}^{*} n^{-3 / 2} \\
= & -\frac{1}{6 s_{n}^{3}} s_{n}^{(3)} H_{2}(t) \phi(t)+\frac{1}{6 s_{n}^{3}} s_{k_{n}}^{(3)} H_{2}(t) \phi(t)-\frac{1}{6 s_{n}^{4}} s_{n}^{(3)} S_{u} H_{3}(t) \phi(t) \\
& -\frac{1}{12 s_{n}^{5}} s_{n}^{(3)}\left(S_{u}^{2}-s_{k_{n}}^{2}\right) H_{4}(t) \phi(t)+\varepsilon_{n}^{*} n^{-3 / 2},
\end{aligned}
$$

where in the last step we use the recurrence relation of Hermite polynomials:

$$
H_{m+1}(t)=t H_{m}(t)-m H_{m-1}(t) .
$$

Noticing that

$$
\begin{aligned}
\kappa_{2, n} & =\frac{1}{72} \frac{\left(s_{n}^{(3)}-s_{k_{n}}^{(3)}\right)^{2}}{\left(s_{n}^{2}-s_{k_{n}}^{2}\right)^{3}}=\frac{1}{72 s_{n}^{6}}\left(s_{n}^{(3)}\right)^{2}+\varepsilon_{n}^{*} n^{-3 / 2}, \\
D_{2}\left(\frac{s_{n} t-S_{u}}{\sqrt{s_{n}^{2}-s_{k_{n}}^{2}}}\right) & =D_{2}(t)+D_{2}^{\prime}(t)\left(\frac{s_{n} t-S_{u}}{\sqrt{s_{n}^{2}-s_{k_{n}}^{2}}}-t\right)+\varepsilon_{n}^{*} n^{-\frac{1}{2}} \\
& =-H_{5}(t) \phi(t)+H_{6}(t) \phi(t)\left(-\frac{1}{s_{n}} S_{u}\right)+\varepsilon_{n}^{*} n^{-1 / 2},
\end{aligned}
$$


we have

$$
\begin{aligned}
& \kappa_{2, n} D_{2}\left(\frac{s_{n} t-S_{u}}{\sqrt{s_{n}^{2}-s_{k_{n}}^{2}}}\right) \\
& =-\frac{1}{72 s_{n}^{6}}\left(s_{n}^{(3)}\right)^{2} H_{5}(t) \phi(t)-\frac{1}{72 s_{n}^{7}}\left(s_{n}^{(3)}\right)^{2} S_{u} H_{6}(t) \phi(t)+\varepsilon_{n}^{*} n^{-3 / 2} .
\end{aligned}
$$

Observing that

$$
\begin{aligned}
\kappa_{3, n} & =\frac{1}{24}\left(s_{n}^{2}-s_{k_{n}}^{2}\right)^{-2} \sum_{j=k_{n}}^{n-1}\left(\sigma_{j}^{(4)}-3\left(\sigma_{j}^{(2)}\right)^{2}\right) \\
& =\frac{1}{24 s_{n}^{4}} \sum_{j=0}^{n-1}\left(\sigma_{j}^{(4)}-3\left(\sigma_{j}^{(2)}\right)^{2}\right)+\varepsilon_{n}^{*} n^{-3 / 2}, \\
D_{3}\left(\frac{s_{n} t-S_{u}}{\sqrt{s_{n}^{2}-s_{k_{n}}^{2}}}\right) & =D_{3}(t)+D_{3}^{\prime}(t)\left(-\frac{1}{s_{n}} S_{u}\right)+\varepsilon_{n}^{*} n^{-1 / 2} \\
& =-H_{3}(t) \phi(t)-\frac{1}{s_{n}} H_{4}(t) \phi(t) S_{u}+\varepsilon_{n}^{*} n^{-1 / 2},
\end{aligned}
$$

we get

$$
\begin{aligned}
\kappa_{3, n} D_{3}\left(\frac{s_{n} t-S_{u}}{\sqrt{s_{n}^{2}-s_{k_{n}}^{2}}}\right)= & -\frac{1}{24 s_{n}^{4}} \sum_{j=0}^{n-1}\left(\sigma_{j}^{(4)}-3\left(\sigma_{j}^{(2)}\right)^{2}\right) H_{3}(t) \phi(t) \\
& -\frac{1}{24 s_{n}^{5}} \sum_{j=0}^{n-1}\left(\sigma_{j}^{(4)}-3\left(\sigma_{j}^{(2)}\right)^{2}\right) H_{4}(t) \phi(t) S_{u}+\varepsilon_{n}^{*} n^{-3 / 2}
\end{aligned}
$$

It is easy to check that

$$
\begin{aligned}
R_{n}\left(\frac{s_{n} t-S_{u}}{\sqrt{s_{n}^{2}-s_{k_{n}}^{2}}}\right)= & -\frac{\left(s_{n}^{(3)}\right)^{3}}{1296 s_{n}^{9}} H_{8}(t) \phi(t)-\frac{1}{120 s_{n}^{5}} \sum_{j=0}^{n-1}\left(\sigma_{j}^{(5)}-10 \sigma_{j}^{(3)} \sigma_{j}^{(2)}\right) H_{4}(t) \phi(t) \\
& -\frac{s_{n}^{(3)}}{144 s_{n}^{7}} \sum_{j=0}^{n-1}\left(\sigma_{j}^{(4)}-3\left(\sigma_{j}^{(2)}\right)^{2}\right) H_{6}(t) \phi(t)+\varepsilon_{n}^{*} n^{-3 / 2} \\
= & \frac{Q_{3, n}(t)}{n^{\frac{3}{2}}}+\varepsilon_{n}^{*} n^{-3 / 2}
\end{aligned}
$$


Plugging the expansions (4.29), (4.30), (4.32), (4.33) and (4.34) into $\mathbb{B}_{n 3}$ defined in (4.20), we deduce that

$$
\begin{gathered}
\mid \mathbb{B}_{n 3}-\left(\Phi(t)+\sum_{\nu=1}^{3} \frac{Q_{\nu, n}(t)}{n^{\nu / 2}}\right) \bar{W}_{k_{n}}+\left(-\frac{1}{s_{n}}\right)\left(\phi(t)+\sum_{\nu=1}^{2} \frac{Q_{\nu, n}^{\prime}(t)}{n^{\nu / 2}}\right) \bar{N}_{1, k_{n}} \\
+\frac{1}{2 !} \frac{1}{s_{n}^{2}}\left(\phi^{\prime}(t)+\frac{Q_{1, n}^{\prime \prime}(t)}{n^{1 / 2}}\right) \bar{N}_{2, k_{n}}+\frac{1}{3 !}\left(-\frac{1}{s_{n}^{3}}\right) \phi^{\prime \prime}(t) \bar{N}_{3, k_{n}} \mid \leq a_{n} \bar{W}_{k_{n}} n^{-\frac{3}{2}} .
\end{gathered}
$$

By using (4.28) and the fact that

$$
0 \leq \bar{W}_{k_{n}} \leq W_{k_{n}} \underset{\text { a.s. }}{\stackrel{n \rightarrow \infty}{\longrightarrow}} W
$$

we obtain the desired (4.23).

The assertion (4.10) follows from (4.21), (4.22) and (4.23), hence the lemma is proved.

Proof of Lemma 4.6. Observe

$$
\bar{W}_{k_{n}}-W=-\frac{1}{\Pi_{k_{n}}} \sum_{u \in \mathbb{T}_{k_{n}}} \mathbf{1}_{\left\{\left|S_{u}\right|>k_{n}\right\}}+\left(W_{k_{n}}-W\right) .
$$

Thus (4.11) follows from (4.24) and the following lemma.

Lemma 4.9 ([26]). Assume the condition (2.1). Then

$$
W-W_{n}=o\left(n^{-\lambda}\right) \quad \text { a.s. }
$$

Similarly, (4.12), (4.13), (4.14) follow from Theorem 2.1 and the following results:

$$
\begin{aligned}
& n \frac{1}{\Pi_{k_{n}}} \sum_{u \in \mathbb{T}_{k_{n}}} S_{u} \mathbf{1}_{\left\{\left|S_{u}\right|>k_{n}\right\}} \stackrel{n \rightarrow \infty}{\longrightarrow} 0 \quad \text { a.s.; } \\
& n^{1 / 2} \frac{1}{\Pi_{k_{n}}} \sum_{u \in \mathbb{T}_{k_{n}}}\left(S_{u}^{2}-s_{k_{n}}^{2}\right) \mathbf{1}_{\left\{\left|S_{u}\right|>k_{n}\right\}} \stackrel{n \rightarrow \infty}{\longrightarrow} 0 \quad \text { a.s., } \\
& \frac{1}{\Pi_{k_{n}}} \sum_{u \in \mathbb{T}_{k_{n}}}\left(S_{u}^{3}-3 S_{u} s_{k_{n}}^{2}-s_{k_{n}}^{(3)}\right) \mathbf{1}_{\left\{\left|S_{u}\right|>k_{n}\right\}} \stackrel{n \rightarrow \infty}{\longrightarrow} 0 \quad \text { a.s., }
\end{aligned}
$$

which can be easily proved by following the lines of the proof of (4.24). 


\section{Convergence rates of the relevant martingales}

In this section, we shall prove Theorem 2.1. Recall that we assume throughout the article that $l_{n}=0$. Then the martingales reduce to the following simplified versions:

$$
\begin{aligned}
& N_{1, n}=\frac{1}{\Pi_{n}} \sum_{u \in \mathbb{T}_{n}} S_{u} ; \\
& N_{2, n}=\frac{1}{\Pi_{n}} \sum_{u \in \mathbb{T}_{n}}\left(S_{u}^{2}-s_{n}^{2}\right) ; \\
& N_{3, n}=\frac{1}{\Pi_{n}} \sum_{u \in \mathbb{T}_{n}}\left(S_{u}^{3}-3 S_{u} s_{n}^{2}-s_{n}^{(3)}\right) .
\end{aligned}
$$

It is easy to verify that they are martingales with respect to the filtration $\mathscr{D}_{n}$, and we omit the details (see [17]).

We shall only offer the detailed proof of part (3), as parts (1) and (2) will follow by the same way with minor changes.

The proof is adapted from Asmussen [1]. The key idea is to find a proper truncation to show the convergence of the series $\sum_{n} a_{n}\left(N_{3, n+1}-N_{3, n}\right)$ with suitable $a_{n}$, which gives the information on the convergence rate of $\sum_{n=\kappa}^{\infty} N_{3, n}$. The proof relies on the following lemma.

Lemma 5.1 ([1], Lemma 2). Let $\left\{\alpha_{n}, \beta_{n}, n \geq 1\right\}$ be sequences of real numbers. If $0<\alpha_{n} \nearrow \infty$, and the series $\sum_{n=1}^{\infty} \alpha_{n} \beta_{n}$ converges, then

$$
\sum_{n=\kappa}^{\infty} \beta_{n}=o\left(\frac{1}{\alpha_{\kappa}}\right) .
$$

Proof of Part (3) in Theorem 2.1. We begin by introducing some notation:

$$
\begin{aligned}
& \lambda_{\delta}=\lambda-3-\delta, \quad X_{u}=S_{u}^{3}-3 S_{u} s_{n}^{2}-s_{n}^{(3)} \quad \text { for } u \in \mathbb{T}_{n}, \\
& \mathrm{I}_{n}=N_{3, n+1}-N_{3, n}=\frac{1}{\Pi_{n}} \sum_{u \in \mathbb{T}_{n}}\left(\frac{1}{m_{n}} \sum_{i=1}^{N_{u}} X_{u i}-X_{u}\right) \\
& \mathrm{I}_{n}^{\prime}=\frac{1}{\Pi_{n}} \sum_{u \in \mathbb{T}_{n}}\left(\frac{1}{m_{n}} \sum_{i=1}^{N_{u}} X_{u i}-X_{u}\right) \mathbf{1}_{\left\{N_{u} \leq \Pi_{n} / n^{\lambda} \delta\right\}} .
\end{aligned}
$$

If we can prove that the series

$$
\sum_{n=1}^{\infty} n^{\lambda_{\delta}} \mathrm{I}_{n} \quad \text { converges a.s. }
$$

then by setting $V_{3}=\sum_{n=1}^{\infty} \mathrm{I}_{n}+\mathrm{I}_{1}$ and using Lemma 5.1, we obtain the desired conclusion. 
We prove (5.1) by showing the following three series converge:

$$
\sum_{n=1}^{\infty} n^{\lambda_{\delta}}\left(\mathrm{I}_{n}-\mathrm{I}_{n}^{\prime}\right), \quad \sum_{n=1}^{\infty} n^{\lambda_{\delta}}\left(\mathrm{I}_{n}^{\prime}-\mathbb{E}_{\xi, n} \mathrm{I}_{n}^{\prime}\right), \quad \sum_{n=1}^{\infty} n^{\lambda_{\delta}} \mathbb{E}_{\xi, n} \mathrm{I}_{n}^{\prime} .
$$

By using an inequality for moment of sums of independent random variables with mean zero, it is easy to see that for $\widehat{S}_{n}=\sum_{j=0}^{n-1} \widehat{L}_{j}$,

$$
\mathbb{E}_{\xi}\left|\widehat{S}_{n}\right|^{r} \leq n^{\frac{r}{2}-1} \sum_{j=0}^{n-1} \mathbb{E}_{\xi}\left|\widehat{L}_{j}\right|^{r} \leq K_{\xi} n^{\frac{r}{2}}
$$

whence for $|u|=n$,

$$
\mathbb{E}_{\xi}\left|X_{u}\right| \leq K_{\xi} n^{3 / 2} ; \quad \mathbb{E}_{\xi}\left|X_{u}\right|^{2} \leq K_{\xi} n^{3} .
$$

For the first series in (5.2), we observe that

$$
\begin{aligned}
\mathbb{E}_{\xi}\left|I_{n}-I_{n}^{\prime}\right| & \leq \frac{1}{\Pi_{n}} \mathbb{E}_{\xi} \sum_{u \in \mathbb{T}_{n}}\left|\frac{1}{m_{n}} \sum_{i=1}^{N_{u}} X_{u i}-X_{u}\right| \mathbf{1}_{\left\{N_{u} / m_{n}>n^{-\lambda} \delta \Pi_{n}\right\}} \\
& \leq \frac{K_{\xi} n^{3}}{\Pi_{n}} \mathbb{E}_{\xi} \sum_{u \in \mathbb{T}_{n}}\left(N_{u} / m_{n}+1\right) \mathbf{1}_{\left\{N_{u} / m_{n}>n^{-\lambda} \delta \Pi_{n}\right\}} \\
& =K_{\xi} n^{3} \mathbb{E}_{\xi}\left(\widehat{N}_{n} / m_{n}+1\right) \mathbf{1}_{\left\{\widehat{N}_{n} / m_{n}>n^{-\lambda_{\delta}} \Pi_{n}\right\}} \\
& \leq K_{\xi} n^{3} \frac{1}{\ln ^{1+\lambda}\left(\Pi_{n} / n^{\lambda_{\delta}}\right)} \mathbb{E}_{\xi}\left(\widehat{N}_{n} / m_{n}+1\right) \ln ^{1+\lambda}\left(\widehat{N}_{n} / m_{n}\right) \\
& \leq(4.18) K_{\xi} n^{2-\lambda} \mathbb{E}_{\xi}\left(\widehat{N}_{n} / m_{n}+1\right)\left(\ln ^{+} \widehat{N}_{n}\right)^{1+\lambda}+K_{\xi} n^{2-\lambda}\left(\ln ^{-} m_{n}\right)^{1+\lambda}
\end{aligned}
$$

We see that

$$
\begin{aligned}
& \mathbb{E} \sum_{n=1}^{\infty} n^{\lambda_{\delta}+2-\lambda}\left[\mathbb{E}_{\xi}\left(\widehat{N}_{n} / m_{n}+1\right)\left(\ln ^{+} \widehat{N}_{n}\right)^{1+\lambda}+\left(\ln ^{-} m_{n}\right)^{1+\lambda}\right] \\
& \quad=\sum_{n=1}^{\infty} n^{-1-\delta}\left[\mathbb{E}\left(\widehat{N}_{0} / m_{0}+1\right)\left(\ln ^{+} \widehat{N}_{0}\right)^{1+\lambda}+\mathbb{E}\left(\ln ^{-} m_{0}\right)^{1+\lambda}\right]<\infty
\end{aligned}
$$

which implies that

$$
\sum_{n=1}^{\infty} n^{\lambda_{\delta}+2-\lambda}\left[\mathbb{E}_{\xi}\left(\widehat{N}_{n} / m_{n}+1\right)\left(\ln ^{+} \widehat{N}_{n}\right)^{1+\lambda}+\left(\ln ^{-} m_{n}\right)^{1+\lambda}\right]<\infty \quad \text { a.s. }
$$


Thus

$$
\begin{aligned}
\mathbb{E}_{\xi}\left|\sum_{n=1}^{\infty} n^{\lambda_{\delta}}\left(\mathrm{I}_{n}-\mathrm{I}_{n}^{\prime}\right)\right| & \leq \sum_{n=1}^{\infty} n^{\lambda_{\delta}} \mathbb{E}_{\xi}\left|\mathrm{I}_{n}-\mathrm{I}_{n}^{\prime}\right|<\infty, \\
\mathbb{E}_{\xi}\left|\sum_{n=1}^{\infty} n^{\lambda_{\delta}} \mathbb{E}_{\xi, n} \mathrm{I}_{n}^{\prime}\right| & =\mathbb{E}_{\xi}\left|\sum_{n=1}^{\infty} n^{\lambda_{\delta}} \mathbb{E}_{\xi, n}\left(\mathrm{I}_{n}-\mathrm{I}_{n}^{\prime}\right)\right| \leq \sum_{n=1}^{\infty} n^{\lambda_{\delta}} \mathbb{E}_{\xi}\left|\mathrm{I}_{n}-\mathrm{I}_{n}^{\prime}\right|<\infty .
\end{aligned}
$$

It follows that the series $\sum_{n=1}^{\infty} n^{\lambda_{\delta}}\left(\mathrm{I}_{n}-\mathrm{I}_{n}^{\prime}\right)$ and $\sum_{n=1}^{\infty} n^{\lambda_{\delta}} \mathbb{E}_{\xi, n} \mathrm{I}_{n}^{\prime}$ converge a.s.

It remains to prove the a.s. convergence of $\sum_{n=1}^{\infty} n^{\lambda_{\delta}}\left(\mathrm{I}_{n}^{\prime}-\mathbb{E}_{\xi, n} \mathrm{I}_{n}^{\prime}\right)$. By using the fact that $\sum_{k=1}^{n} k^{\lambda_{\delta}}\left(\mathrm{I}_{k}^{\prime}-\mathbb{E}_{\xi, k} \mathrm{I}_{k}^{\prime}\right)$ is a martingale with respect to $\left\{\mathscr{D}_{n+1}\right\}$ and by the a.s. convergence of an $L^{2}$ bounded martingale (see, e.g., [15], page 251, Example 4.9), we only need to show that the series

$$
\sum_{n=1}^{\infty} n^{2 \lambda_{\delta}} \mathbb{E}_{\xi}\left(\mathrm{I}_{n}^{\prime}-\mathbb{E}_{\xi, n} \mathrm{I}_{n}^{\prime}\right)^{2} \quad \text { converges a.s. }
$$

To this end, we first note that

$$
\begin{aligned}
\mathbb{E}_{\xi} & {\left[\left(\frac{1}{m_{n}} \sum_{i=1}^{N_{u}} X_{u i}-X_{u}\right)^{2} \mathbf{1}_{\left\{N_{u} / m_{n} \leq n^{-\lambda_{\delta}} \Pi_{n}\right\}} \mid \mathcal{F}_{n}\right] } \\
= & \mathbb{E}_{\xi}\left\{\mathbb{E}_{\xi}\left[\left(\frac{1}{m_{n}} \sum_{i=1}^{N_{u}} X_{u i}-X_{u}\right)^{2} \mid N_{u}\right] \mathbf{1}_{\left\{N_{u} / m_{n} \leq n^{-\lambda_{\delta}} \Pi_{n}\right\}}\right\} \\
\leq & \mathbb{E}_{\xi}\left\{2\left(N_{u} \sum_{i=1}^{N_{u}} \frac{\mathbb{E}_{\xi} X_{u i}^{2}}{m_{n}^{2}}+\mathbb{E}_{\xi} X_{u}^{2}\right) \mathbf{1}_{\left\{N_{u} / m_{n} \leq n^{-\lambda_{\delta}} \Pi_{n}\right\}}\right\} \\
\leq(5.4) & K_{\xi} n^{3}\left(\mathbb{E}_{\xi} \frac{N_{u}^{2}}{m_{n}^{2}} \mathbf{1}_{\left\{N_{u} / m_{n} \leq n^{-\lambda_{\delta}} \Pi_{n}\right\}}+1\right) \\
& =K_{\xi} n^{3}\left(\mathbb{E}_{\xi} \frac{\widehat{N}_{n}^{2}}{m_{n}^{2}} \mathbf{1}_{\left\{\widehat{N}_{n} / m_{n} \leq n^{-\lambda_{\delta}} \Pi_{n}\right\}}+1\right) .
\end{aligned}
$$

We next observe that

$$
\begin{aligned}
n^{2 \lambda_{\delta}} & \mathbb{E}_{\xi}\left(\mathrm{I}_{n}^{\prime}-\mathbb{E}_{\xi, n} \mathrm{I}_{n}^{\prime}\right)^{2} \\
= & \frac{n^{2 \lambda_{\delta}}}{\Pi_{n}^{2}} \mathbb{E}_{\xi} \sum_{u \in \mathbb{T}_{n}} \mathbb{E}_{\xi, n}\left[\left(\frac{1}{m_{n}} \sum_{i=1}^{N_{u}} X_{u i}-X_{u}\right) \mathbf{1}_{\left\{N_{u} / m_{n} \leq n^{-\lambda_{\delta}} \Pi_{n}\right\}}\right. \\
& \left.-\mathbb{E}_{\xi, n}\left(\frac{1}{m_{n}} \sum_{i=1}^{N_{u}} X_{u i}-X_{u}\right) \mathbf{1}_{\left\{N_{u} / m_{n} \leq n^{-\lambda_{\delta}} \Pi_{n}\right\}}\right]^{2}
\end{aligned}
$$




$$
\begin{aligned}
& \left.\leq \frac{n^{2 \lambda_{\delta}}}{\Pi_{n}^{2}} \mathbb{E}_{\xi} \sum_{u \in \mathbb{T}_{n}} \mathbb{E}_{\xi, n}\left[\left(\frac{1}{m_{n}} \sum_{i=1}^{N_{u}} X_{u i}-X_{u}\right) \mathbf{1}_{\left\{N_{u} / m_{n} \leq n^{-\lambda} \delta\right.} \Pi_{n}\right\}\right]^{2} \\
& =\frac{n^{2 \lambda_{\delta}}}{\Pi_{n}^{2}} \mathbb{E}_{\xi} \sum_{u \in \mathbb{T}_{n}} \mathbb{E}_{\xi}\left[\left(\frac{1}{m_{n}} \sum_{i=1}^{N_{u}} X_{u i}-X_{u}\right)^{2} \mathbf{1}_{\left\{N_{u} / m_{n} \leq n^{-\lambda_{\delta}} \Pi_{n}\right\}} \mid \mathcal{F}_{n}\right] \\
& \leq \frac{K_{\xi} n^{3+2 \lambda_{\delta}}}{\Pi_{n}} \mathbb{E}_{\xi} \frac{\widehat{N}_{n}^{2}}{m_{n}^{2}} \mathbf{1}_{\left\{\widehat{N}_{n} / m_{n} \leq n^{-\lambda_{\delta}} \Pi_{n}\right\}}+\frac{K_{\xi} n^{3+2 \lambda_{\delta}}}{\Pi_{n}} \\
& =\frac{K_{\xi} n^{3+2 \lambda_{\delta}}}{\Pi_{n}} \mathbb{E}_{\xi} \frac{\widehat{N}_{n}^{2}}{m_{n}^{2}}\left(\mathbf{1}_{\left\{\widehat{N}_{n} / m_{n} \leq e^{2 \lambda}\right\}}+\mathbf{1}_{\left\{e^{2 \lambda}<\widehat{N}_{n} / m_{n} \leq n^{-\lambda_{\delta}} \Pi_{n}\right\}}\right)+\frac{K_{\xi} n^{3+2 \lambda_{\delta}}}{\Pi_{n}} \\
& \leq \frac{K_{\xi} n^{3+2 \lambda_{\delta}}}{\Pi_{n}} \mathbb{E}_{\xi} \frac{\widehat{N}_{n}^{2}}{m_{n}^{2}} \mathbf{1}_{\left\{e^{2 \lambda}<\widehat{N}_{n} / m_{n} \leq n^{-\lambda_{\delta}} \Pi_{n}\right\}}+\frac{K_{\xi} n^{3+2 \lambda_{\delta}}}{\Pi_{n}} \\
& \leq \frac{K_{\xi} n^{3+2 \lambda_{\delta}}}{\Pi_{n}} \frac{\Pi_{n}}{n^{\lambda_{\delta}}}\left(\ln \frac{\Pi_{n}}{n^{\lambda_{\delta}}}\right)^{-1-\lambda} \mathbb{E}_{\xi} \frac{\widehat{N}_{n}^{2}}{m_{n}^{2}}\left[\frac{\widehat{N}_{n}}{m_{n}}\left(\ln ^{+} \frac{\widehat{N}_{n}}{m_{n}}\right)^{-1-\lambda}\right]^{-1}+\frac{K_{\xi} n^{3+2 \lambda_{\delta}}}{\Pi_{n}} \\
& \text { (because } x(\ln x)^{-1-\lambda} \text { is increasing for } x>e^{2 \lambda} \text { ) } \\
& \leq K_{\xi} n^{2+\lambda_{\delta}-\lambda}\left(\mathbb{E}_{\xi} \frac{\widehat{N}_{n}}{m_{n}}\left(\ln ^{+} \widehat{N}_{n}\right)^{1+\lambda}+\left(\ln ^{-} m_{n}\right)^{1+\lambda}\right)+\frac{K_{\xi} n^{3+2 \lambda_{\delta}}}{\Pi_{n}} \text {. }
\end{aligned}
$$

By the above estimates and (5.5), we see that the series $\sum_{n=1}^{\infty} n^{2 \lambda_{\delta}} \mathbb{E}_{\xi}\left(\mathrm{I}_{n}^{\prime}-\mathbb{E}_{\xi, n} \mathrm{I}_{n}^{\prime}\right)^{2}$ converges a.s.

So we have proved the three series in (5.2) converges a.s. and hence (5.1) holds. By setting $V_{3}=\sum_{n=1}^{\infty} \mathrm{I}_{n}+N_{3,1}$, we have $N_{3, n}-V_{3}=\sum_{j=n}^{\infty} \mathrm{I}_{j}$, and hence part (3) of the lemma follows from Lemma 5.1 and (5.1).

\section{Acknowledgements}

We are grateful to the editor in charge of the article and to two anonymous referees for their very valuable comments, remarks or suggestions, which significantly contributed to improving the quality of the article. The work has been partially supported by the National Natural Science Foundation of China (Grants No. 11101039, No. 11271045, No. 11571052, No. 11401590), by the Fundamental Research Funds for the Central Universities (China), and by the Natural Science Foundation of Guangdong Province (China), Grant no. 2015A030313628. Q. Liu has also benefited from a delegation CNRS (France) during the preparation of the article.

\section{References}

[1] Asmussen, S. (1976). Convergence rates for branching processes. Ann. Probab. 4 139-146. MR0391286 
[2] Asmussen, S. and Kaplan, N. (1976). Branching random walks. I. Stochastic Process. Appl. 4 1-13. MR0400429

[3] Athreya, K.B. and Karlin, S. (1971). On branching processes with random environments. I. Extinction probabilities. Ann. Math. Stat. 42 1499-1520. MR0298780

[4] Athreya, K.B. and Karlin, S. (1971). Branching processes with random environments. II. Limit theorems. Ann. Math. Stat. 42 1843-1858. MR0298781

[5] Bai, Z.D. and Zhao, L.C. (1986). Edgeworth expansions of distribution functions of independent random variables. Sci. Sinica Ser. A 29 1-22. MR0851973

[6] Baillon, J.-B., Clément, Ph., Greven, A. and den Hollander, F. (1993). A variational approach to branching random walk in random environment. Ann. Probab. 21 290-317. MR1207227

[7] Biggins, J.D. (1990). The central limit theorem for the supercritical branching random walk, and related results. Stochastic Process. Appl. 34 255-274. MR1047646

[8] Biggins, J.D. and Kyprianou, A.E. (2004). Measure change in multitype branching. Adv. in Appl. Probab. 36 544-581. MR2058149

[9] Birkner, M., Geiger, J. and Kersting, G. (2005). Branching processes in random environment a view on critical and subcritical cases. In Interacting Stochastic Systems 269-291. Berlin: Springer. MR2118578

[10] Bovier, A. and Hartung, L. (2015). Variable speed branching Brownian motion 1. Extremal processes in the weak correlation regime. ALEA Lat. Am. J. Probab. Math. Stat. 12 261-291. MR3351476

[11] Chen, X. (2001). Exact convergence rates for the distribution of particles in branching random walks. Ann. Appl. Probab. 11 1242-1262. MR1878297

[12] Comets, F. and Popov, S. (2007). On multidimensional branching random walks in random environment. Ann. Probab. 35 68-114. MR2303944

[13] Comets, F. and Popov, S. (2007). Shape and local growth for multidimensional branching random walks in random environment. ALEA Lat. Am. J. Probab. Math. Stat. 3 273-299. MR2365644

[14] Comets, F. and Yoshida, N. (2011). Branching random walks in space-time random environment: Survival probability, global and local growth rates. J. Theoret. Probab. 24 657-687. MR2822477

[15] Durrett, R. (1996). Probability: Theory and Examples, 2nd ed. Belmont, CA: Duxbury Press. MR1609153

[16] Fang, M. and Zeitouni, O. (2012). Branching random walks in time inhomogeneous environments. Electron. J. Probab. 17 no. 67, 18. MR2968674

[17] Gao, Z. and Liu, Q. (2016). Exact convergence rates in central limit theorems for a branching random walk with a random environment in time. Stochastic Process. Appl. 126 2634-2664. MR3522296

[18] Gao, Z. and Liu, Q. (2016). First- and second-order expansions in the central limit theorem for a branching random walk. C. R. Math. Acad. Sci. Paris 354 532-537. MR3488125

[19] Gao, Z., Liu, Q. and Wang, H. (2014). Central limit theorems for a branching random walk with a random environment in time. Acta Math. Sci. Ser. B Engl. Ed. 34 501-512. MR3174096

[20] Gao, Z. (2016). Exact convergence rate of the local limit theorem for branching random walks on the integer lattice. Stochastic Process. Appl. To appear.

[21] Greven, A. and den Hollander, F. (1992). Branching random walk in random environment: Phase transitions for local and global growth rates. Probab. Theory Related Fields 91 195-249. MR1147615

[22] Grübel, R. and Kabluchko, Z. (2016). Edgeworth expansions for profiles of lattice branching random walks. Ann. Inst. Henri Poincaré Probab. Stat. To appear. Available at arXiv:1503.04616.

[23] Harris, T.E. (1963). The Theory of Branching Processes. Die Grundlehren der Mathematischen Wissenschaften 119. Berlin: Springer. MR0163361

[24] Hu, Y. and Yoshida, N. (2009). Localization for branching random walks in random environment. Stochastic Process. Appl. 119 1632-1651. MR2513122 
[25] Huang, C., Liang, X. and Liu, Q. (2014). Branching random walks with random environments in time. Front. Math. China 9 835-842. MR3240345

[26] Huang, C. and Liu, Q. (2014). Convergence rates for a supercritical branching process in a random environment. Markov Process. Related Fields 20 265-286. MR3241526

[27] Joffe, A. and Moncayo, A.R. (1973). Random variables, trees, and branching random walks. Adv. Math. 10 401-416. MR0319283

[28] Kabluchko, Z. (2012). Distribution of levels in high-dimensional random landscapes. Ann. Appl. Probab. 22 337-362. MR2932549

[29] Kaplan, N. and Asmussen, S. (1976). Branching random walks. II. Stochastic Process. Appl. 4 15-31. MR0400430

[30] Klebaner, C.F. (1982). Branching random walk in varying environments. Adv. in Appl. Probab. 14 359-367. MR0650128

[31] Liang, X. and Liu, Q. (2013). Weighted moments of the limit of a branching process in a random environment. Tr. Mat. Inst. Steklova 282 135-153. MR3308588

[32] Liu, Q. (2007). Branching random walks in random environment. In Proceedings of the 4th International Congress of Chinese Mathematicians (ICCM 2007) (L. Ji, K. Liu, L. Yang and S.T. Yau, eds.) II 702-719.

[33] Nakashima, M. (2011). Almost sure central limit theorem for branching random walks in random environment. Ann. Appl. Probab. 21 351-373. MR2759206

[34] Petrov, V.V. (1975). Sums of Independent Random Variables. New York: Springer. MR0388499

[35] Révész, P. (1994). Random Walks of Infinitely Many Particles. River Edge, NJ: World Scientific. MR1645302

[36] Révész, P., Rosen, J. and Shi, Z. (2005). Large-time asymptotics for the density of a branching Wiener process. J. Appl. Probab. 42 1081-1094. MR2203824

[37] Shi, Z. (2015). Branching Random Walks. Lecture Notes in Math. 2151. Cham: Springer. MR3444654

[38] Smith, W.L. and Wilkinson, W.E. (1969). On branching processes in random environments. Ann. Math. Stat. 40 814-827. MR0246380

[39] Stam, A.J. (1966). On a conjecture by Harris. Z. Wahrsch. Verw. Gebiete 5 202-206. MR0202201

[40] Tanny, D. (1988). A necessary and sufficient condition for a branching process in a random environment to grow like the product of its means. Stochastic Process. Appl. 28 123-139. MR0936379

[41] Wang, X. and Huang, C. (2016). Convergence of martingale and moderate deviations for a branching random walk with a random environment in time. J. Theoret. Probab. To appear.

[42] Yoshida, N. (2008). Central limit theorem for branching random walks in random environment. Ann. Appl. Probab. 18 1619-1635. MR2434183

[43] Zeitouni, O. (2012). Branching random walks and Gaussian fields. Notes for Lectures.

Received September 2015 and revised May 2016 